$$
\begin{aligned}
\frac{\mu_{1}}{c} & =\sum_{i}^{6} M_{1 n} \varrho_{n} \\
\frac{\mu_{2}}{c} & =\sum_{1}^{6} M_{2 n} \varrho_{n} \\
\frac{\mu_{3}}{c} & =\sum_{1}^{6} M_{3 n} \varrho_{n} \\
\frac{\mu_{4}}{c} & =\sum_{1}^{6} M_{4 n} \varrho_{n} \\
\frac{\mu_{5}}{c} & =\sum_{1}^{6} M_{5 n} \varrho_{n}
\end{aligned}
$$

$$
\left.\begin{array}{rl}
-\frac{m_{1}}{c} & =\sum_{1}^{6} M_{1 n} r_{n} \\
-\frac{m_{2}}{c} & =\sum_{1}^{6} M_{2 n} r_{n} \\
-\frac{m_{3}}{c} & =\sum_{1}^{6} M_{3 n} r_{n} \\
-\frac{m_{1}}{c} & =\sum_{1}^{6} M_{4 n} r_{n} \\
-\frac{m_{5}}{c} & =\sum_{1}^{6} M_{5 n} r_{6}
\end{array}\right\}
$$

Bisher haben wir uns unsere Arbeit durch keine Vernachlässigung erleichtert. Das wollen wir jetzt tun, indem wir den Abstand der Leiter 3 und 4 als sehr groß voraussetzen. Dann kann man die Gegeninduktivität irgendeines Leiters 4, 5, 6 mit den Leiterpaaren I und 2 und umgekehrt die Gegeninduktivität irgendeines Leiters I, 2, 3 mit den Leiterpaaren 4 und 5 gleich Null setzen. Die Forderung 49) zerfällt dann in die beiden Forderungen $I$ a) tund $I b$ ).

Der dann noch übrigbleibende Rest der Gl. 5o) und 5I) wird recht übersichtlich und lautet, wenn wir noch

setzen:

$$
\mathrm{M}_{11}=\mathrm{a} ; \quad \mathrm{M}_{12}=-\mathrm{a} ; \quad \mathrm{M}_{13}=-\mathrm{M}_{21}=-\mathrm{b}
$$

$$
\left.\begin{array}{c}
\frac{\mu_{1}}{\mathrm{c}}=\mathrm{a} \varrho_{1}-\mathrm{a} \varrho_{2}-\mathrm{b} \varrho_{3} \\
\frac{\mu_{2}}{\mathrm{c}}=\mathrm{b} \varrho_{1}+\mathrm{a} \varrho_{2}-\mathrm{a} \varrho_{3} \\
\frac{\mu_{4}}{\mathrm{c}}=\mathrm{a} \varrho_{4}-\mathrm{a} \varrho_{5}-\mathrm{b} \varrho_{6} \\
\frac{\mu_{5}}{\mathrm{c}}=\mathrm{b} \varrho_{4}+\mathrm{a} \varrho_{5}-\mathrm{a} \varrho_{6}
\end{array}\right\}
$$

Hieraus folgen die angegebenen Bezichungen 8a) und durch Änderung des Vorzeichens die, Beziehungen $8 \mathrm{~b}$ ).

\title{
Einschaltspannungen der Spule aus zwei Windungen.
}

$$
\text { Von }
$$

Ludwig Dreyfus, Berlin-Niederschönhausen.

Das Studium der Fortpflanzung von Wanderwellen durch Spulen ist in jüngster Zeit in neue Bahnen gelenkt worden. Während man sich ursprünglich bemühte, die vieldrähtige Spule durch einen Ersatzstromkreis aus konzentrierten Induktivitäten und Kapazitäten abzubilden, packte Rogowski') das Problem mit glücklichem Griff am

1) Rogowski: „Spulen und Wanderwellen: I. Einschaltungen der Spule aus zwei Windungen": Archiv f. Elektrot. I9I8, 6, S. 265. "II. Resonanzspannungen beim Einschalten einer Spule aus zwei Windungen": Archiv f. Elektrot. 1918, 6, S. 377. "Die Spule bei Wechselstrom": Archiv f. Elektrot. 1918, 7, S. I7. 
anderen Ende an: Er ging von der Spule mit wenigen - zunächst nur zwei - Windungen aus, berücksichtigte aber dafür die verteilte gegenseitige Induktivität und Kapazität der einzelnen Windungen. (Die Kapazität gegen Erde wird vernachlässigt.)

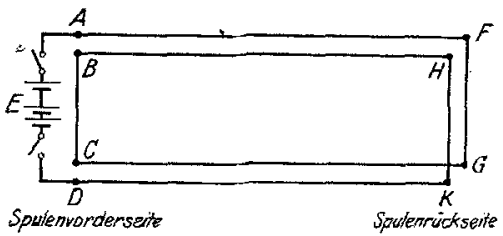

Abb. I. Rogowskis Modell einer Spule aus zwei Windungen.

Das Rogowskische Modell der Spule aus zwei

Windungen zeigt Abb. I. Rogowski betrachtet sonach die Spule als zwei ineinandergeschobene Doppelleitungen AFGC und BHKD. Ihre magnetischen und elektrischen Felder sind in gleicher Art miteinander verkettet, denn die Niveaulinien des elektrischen Feldes sind zugleich Kraftlinien des magnetischen Feldes. Die rechnerischen Grundlagen für diese Betrachtungsweise hat K. W. Wag. ner ${ }^{1}$ ) in einem mehrere Jahre früher erschienenen Aufsatz zusammengestellt.

Die Behandlung der Spule aus zwei Windungen hat Rogowski zu schönen Ergebnissen bezüglich ihres Verhaltens bei hochfrequentem Wechselstrom geführt. Dagegen sind seine Ausführungen über die Einschaltspannungen der Spule aus zwei Windungen nicht leicht auf praktische Fälle zu übertragen. Das hat drei Gründe:

Einmal ist die Spule aus zwei Windungen noch ein gutes Stück von der vieldrähtigen Spule entfernt. Doch war fürs erste die Beschränkung auf zwei Windungen notwendig und ich erblicke sogar das Hauptverdienst der Rogowskischen Arbeiten darin, daß er ungeachtet dieses Abstandes das Problem erstmalig von dieser Seite in Angriff genommen hat.

Schwerwiegend erscheint mir jedoch ein zweites Bedenken: Man kann bei Schaltprozessen nicht darauf rechnen, daß beide Schaltmesser genau gleichzeitig an die Wicklungsenden gelegt werden. Hierin besteht aber die Voraussetzung für die vollkommene oder doch annähernde Symmetrie des elektrischen und magnetischen Feldes gegen die Spulen-Mittelebene und gerade dieser Annahme verdankt Rogowski seine einfachen Ergebnisse. Fällt diese Voraussetzung fort, so muß man auch die Kapazität der Windungen gegen Erde berücksichtigen.

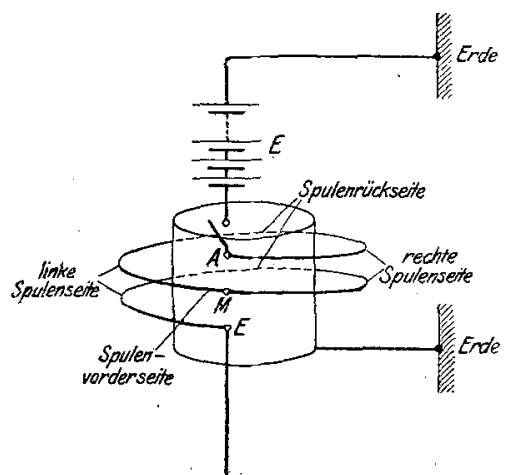

Abb. 2. Modell einer Spule aus zwei Witdungen mit angeschlossener Leitung.

Endlich ist mir kein praktisch wichtiger Schaltprozeß bekannt, bei dem die Einschaltewellen an einem Wicklungsende den Einschaltvorgang am anderen Wicklungsende so entscheidend beeinflussen, wie dies unter den Annahmen der Rogowskischen Arbeit der Fall ist ${ }^{2}$ ).

Die beiden letzten Erwägungen veranlaßten mich, das Rogowskische Modell so abzuändern, wie es Abb. 2 zeigt: Eine widerstandslose Spule aus zwei Windungen sitzt auf einem geerdeten metallischen Kern. Nur der Anfang der Spule-wird plötzlich an Spannung gelegt. Das Spulenende mündet in eine Leitung von beliebigem Wellenwiderstand, die so gefüht sein soll, daB Wanderwellen auf dieser Leitung die Vorgänge in der

1) K. W. Wagner: „Induktionswirkungen von Wanderwellen in Nachbarleitungen : ETZ 1914, S. 639.

2) Nach Einsendung der Arbeit war Herr Rogowski so freundlich, mich auf folgenden Fall aufmerksam zu machen: Man denke sich in Abb. I den ersten Schaiter eingelegt und die Aufladung der Spule vollendet. Nun werde auch der zweite Schalter eingelegt. Für den hierauf folgenden Ausgleichvorgang sind Rogowskis Annahmen erfillt, vorausgesetzt dafie Wanderwellen die Spannungsquelle - als welche auch ein Kabel oder ein Kondensator fungieren kann ohne Verzerrung und Reflexionen ïberbrïcker -können. 
Spule selbst nicht induktiv beeinflussen. Wie in der zitierten Arbeit von Wagner berücksichtigen wir die gegenseitige Kapazität und Induktivität der Windungen, sowie ihre Kapazität gegen Erde. Wir betrachten dabei die Felder als rein transversal, vernachlässigen also die magnetischen Wirkungen des Verschiebungsstromes. Endlich möge der metallische Kern für die Dauer des Ausgleichvorganges als feldfrei angesehen werden. Diese Annahme ist zulässig. Denn auch in sehr fein unterteiltem Eisen erfordert die Ausbildung des magnetischen Feldes ein Vielfaches der Zeit, die eine Wanderwelle braucht, um die Spule zu durchlaufen.

\section{Die einleitenden Schwingungsvorgänge.}

Unter den genannten Voraussetzungen ist es nicht schwer, sich ohne Rechnung ein richtiges Bild von den ersten Schaitwellen zu machen.

(Im folgenden werden die in die Spule hinein (d. h. in der Richtung A-E) laufenden Strom- und Spannungswellen mit $J^{\prime}$ und $V^{\prime}$, die rückläufigen Wellen mit $J^{\prime \prime}$ und $V^{\prime \prime}$ bezeichnet. Die Spannungen und Ströme an den Knotenpunktstellen A, M, E seien $\mathrm{V}$ und J. Arabische Ziffern als Indizes beziehen sich auf die Spulenabschnitte I, 2, 3, die römischen Ziffern geben an, wie viele Wellen seit Beginn des Schaltprozesses bereits in die Wicklung eingedrungen sind. $\left(\mathrm{V}_{1}{ }^{\prime}\right)_{\mathrm{I}}$ ist also die erste Schaltwelle, welche in den Windungsabschnitt $\mathbf{I}$ eindringt.)

Sobald der Schalter geschlossen wird, dringt eine Wanderwelle

$$
\left(V_{1}^{\prime}\right)_{I}=\mathrm{E}
$$

mit Lichtgeschwindigkeit in die erste Windung der Spule ein. Sie wird magnetisch und elektrisch auf die zweite Windung übertragen und breitet sich dort mit Lichtgeschwindigkeit nach beiden Richtungen aus. So entstehen gleichzeitig eine rechtsläufige Welle $\left(\mathrm{V}_{2}{ }^{\prime}\right)_{\mathrm{I}}$ und eine linksläufige Welle $\left(\mathrm{V}_{2}{ }^{\prime \prime}\right)_{\mathrm{I}}$. Die zuletzt genannte Welle induziert ihrerseits die benachbarte Windung 3, so daß auch am Windungsende zwei Wellen entspringen: eine rückläufige Welle $\left(\mathrm{V}_{3}{ }^{\prime \prime}\right)_{\mathrm{I}}$ die mit $\left(\mathrm{V}_{2}{ }^{\prime \prime}\right)_{\mathrm{I}}$ magnetisch und elektrisch gekoppelt ist, und eine vorwärtslaufende Welle $\left(V_{3}{ }^{\prime}\right)_{1}$, welche in die angeschlossene Leitung eindringt. Soweit die Spannungswellen in Betracht kommen, entwickelt sich somit der Einschaltevorgang, wie in Abb. 3 a gezeichnet.

In den Stromwellen der Abb. $3 b$ haben wir nur einen anderen Ausdruck für dieselben Vorgänge zu erblicken. Ist

$$
\mathrm{r}=\sqrt{\overline{\mathrm{L}}}
$$

der Wellenwiderstand der angeschlossenen Leitung, so verbraucht diese bei einer Ladespannung $V_{3 \mathrm{I}}^{\prime}$ den Ladestrom

$$
\mathrm{J}_{3}^{\prime} \mathrm{I}=\frac{\mathrm{V}_{3 \mathrm{I}}^{\prime}}{\mathrm{r}}
$$

Da dieser Strom nur an der Wellenfront $V_{2 \mathrm{~L}}^{\prime \prime}$ der mittleren Windung übertragen werden kann, so haben wir in 2 und 3 zwei linksläufige Wellen $J_{2 \mathrm{I}}^{\prime \prime}$ und $\mathrm{J}_{3 \mathrm{I}}^{\prime \prime}$ entgegengesetzten Vorzeichens anzunehmen. Dabei ist $\int_{2}^{\prime \prime}$ um den zur Erde abfließenden Ladestrom größer als J31. Ähnlich liegen die Verhältnisse zwischen den parallelen Windungsabschnitten I und 2. Die Leitung 2 empfängt ihren Strom $J_{2 I}^{\prime}=J_{2 I}^{\prime \prime}$ an der Wellenfront $V_{1 \mathbf{I}}^{\prime}$ und abermals ist die Stromamplitude $J_{1 \mathrm{I}}^{\prime}$ um den zur Erde abfließenden Ladestrom größer als die Amplitude J'i (vgl. Abb. $3 b^{\prime}$ ).

Je geringer der Abstand zwischen den parallelen Windungsabschnitten, um so geringer sind die Unterschiede zwischen $V_{1}^{\prime}$ und $V_{2}^{\prime}$ einerseits und zwischen $V_{2}^{\prime \prime}$ und $\mathrm{V}_{3}{ }^{\prime \prime}$ andererseits. Im Grenzfall unendlich benachbarter Windungen würde sogar die volle Einschaltespannung $\mathrm{E}$ quer zu den Windungen auf die angeschlossene Leitung 


$=$

Spannungsverteilung.

Abb. 3.

Stromverteilung.

Erstes Intervall $(\mathrm{p}=\mathrm{=}=\mathrm{I})$.

Abb. 3 a.
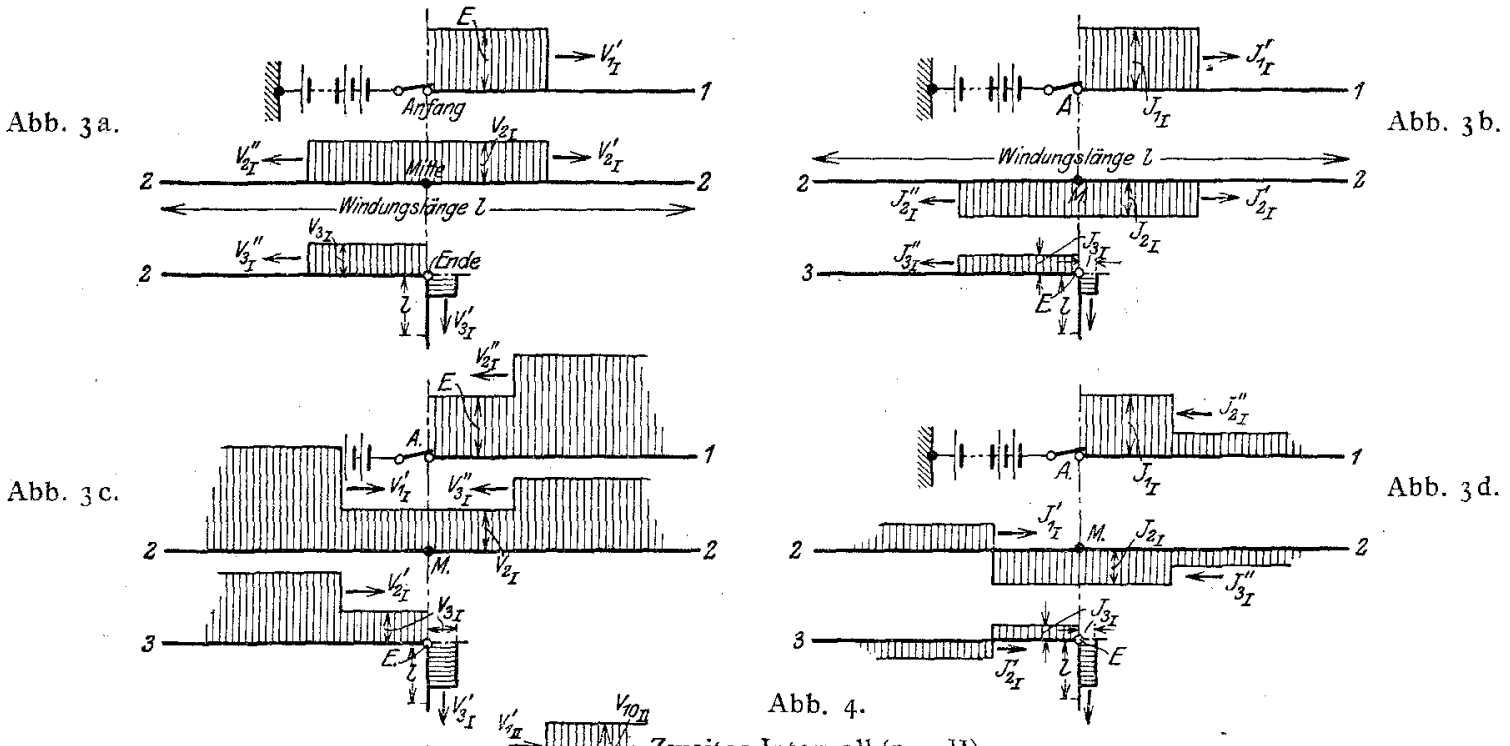

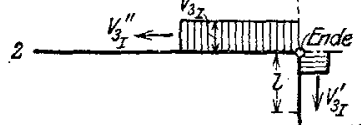
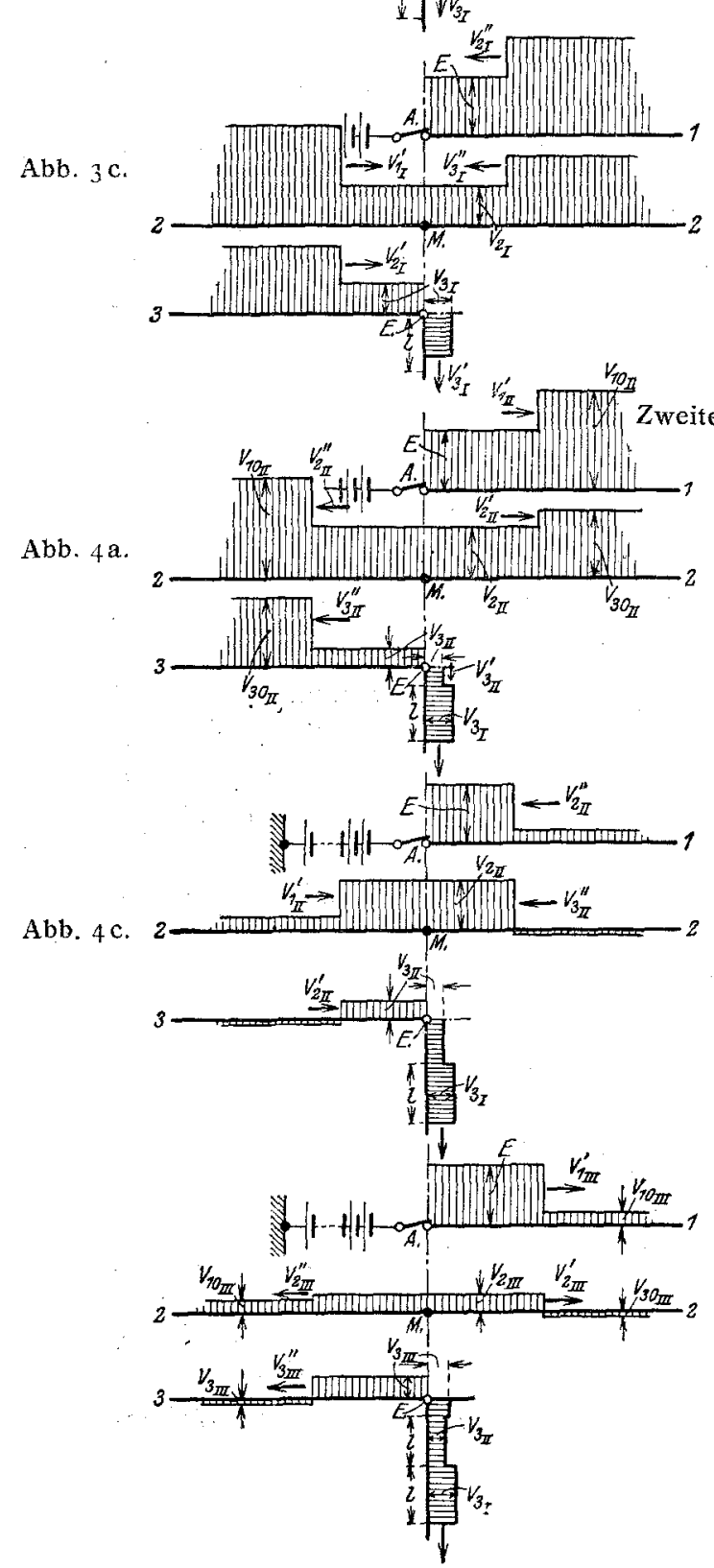

bb. $3 \mathrm{~d}$.

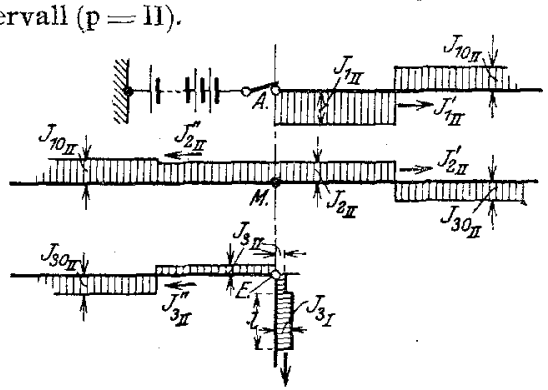

Abb. 4 b.

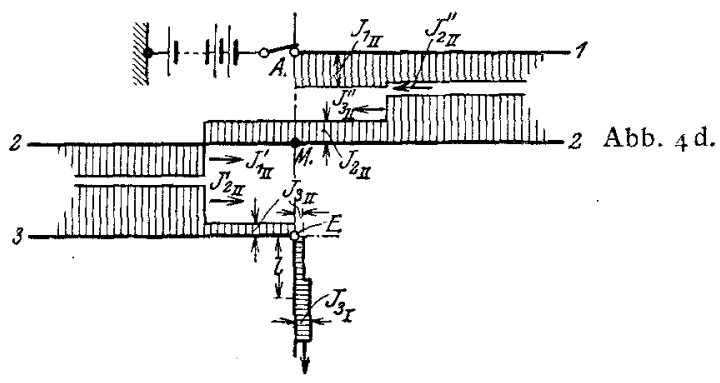

Abb. 5 .

Drittes Intervall $(\mathrm{p}=-\mathrm{III})$.

Abb. 3 bis 5 . Spannungs- und Stromwellen während der ersten Schwingungsintervalle. 
übergehen. Je weiter umgekehrt die Windungen voneinander entfernt sind, um so größere Spannungsdifferenzen sind zwischen ihnen zu erwarten.

Weon die Wellen nahezu eine Windung zurückgelegt haben, wird der Spannungszustand durch Abb. $3 c$ wiedergegeben. Da die Wellen $V_{1 \mathrm{I}}^{\prime}$ und $V_{2 \mathrm{I}}^{\prime \prime}$ einerseits und $V_{21}^{\prime}$ und $V_{31}^{\prime \prime}$ andererseits übereinander weggelaufen sind, so hat sich die Spannung gegen Erde nahezu verdoppelt. Die Windungsspannungen sind jedoch ihrer Größe nicht ihrer Lage nach - unverändert geblieben.

Am Anfang der Spule angelangt, trifft die Welle $V_{2 I}^{\prime \prime}$ auf einen Knotenpunkt A, dessen Potential E vorgeschrieben ist. Sie wird daher total reflektiert und zieht sich als Entladewelle in die Spule zurück. Als einen Knotenpunkt ähnlicher Art haben wir auch die Spulenmitte M zu betrachten. Zwar wird ihr kein festes Potential aufgenötigt. Doch übt die Nachbarschaft des konstanten Potentials in A immerhin einen gewissen Zwang aus, der allzu große Schwankungen verhindert. Es ist daher bei kleinem Windungsabstand die zurückgeworfene Welle $V_{2 \mathrm{II}}^{\prime \prime}$ nur wenig kleiner, als die angekommene Welle $\mathrm{E}$, und ebenso die reflektierte Welle $V_{2 \mathrm{II}}^{\prime}$ nur wenig kleiner, als die auftreffende Welle $V_{31}^{\prime \prime}($ Abb. 4a).

Infolge der elektromagnetischen Koppelung iberträgt sich die Reflexion auch auf den Windungsabschnitt 3, wo eine Entladewelle $V_{31}^{\prime \prime}$ vom Spulenende in die Spule einzieht. Im gewissen Sinne bildet daher auch der Endpunkt der Spule einen Schwingungsknoten. Da er aber von dem starren Knotenpunkt A weiter als die Spulenmitte entfernt ist, ist sein Potential auch größeren Schwankungen unterworfen. Im vorliegenden Falle erniedrigt es sich und dementsprechend dringt eine Entladewelle $V_{3}^{\prime}$ II in die Leitung ein.

Wir verfolgen auch dieses Wellenspiel, bis die zurückgeworfenen Wellen eine Windungslänge zurückgelegt haben. Kurz vor diesem Zeitpunkt wird die Spannungsverteilung durch $\mathrm{Abb} .4 \mathrm{C}$ wiedergegeben: Indem die Entladewellen übereinander weggelaufen sind, haben sie die Spule fast vollkommen entladen. Zwischen Spulenmitte und Spulenende ist die Spannung sogar unter das Null-Niveau (Erde) gesunken. Im nächsten Augenblick wird jedoch die Entladewelle $V_{2 I}^{\prime \prime}$ bei A total reflektiert

$$
\mathrm{V}_{1 \mathrm{III}}^{\prime}=-\mathrm{V}_{2 \mathrm{II}}^{\prime \prime}
$$

und kehrt als Ladewelle in die Spule zurück. Ähnliche Reflexionen erleiden die übrigen Wanderwellen, so daß nun dem Entladevorgang wieder eine Ladeschwingung folgt.

So viel über die Spannungswellen, welche den Ausgleichsvorgang einleiten. Das gleichzeitige Spiel der Stromwellen ist ohne Rechnung schwerer zu übersehen. Es ist in den $\mathrm{Abb}$. $3 \mathrm{~b}$, d und $4 \mathrm{~b}, \mathrm{~d}$ nach später zu erörternden Formeln aufgezeichnet worden.

\section{Berechnung der Strom- und Spannungswellen für beliebige Anfangs- bedingungen.}

Wie die besprochenen Abbildungen zeigen, läßt jede Welle nach Durchlaufen der vollen Windungslänge einen Ladezustand auf der Spule zurück, welcher der folgenden refiektierten Welle gewisse Anfangsbedingungen auferlegt. Wir charakterisieren diesen Anfangszustand durch die Potentiale $\left(V_{10}\right)_{p}$ und $\left(V_{30}\right)_{p}$, die vor dem Eindringen der p-ten Schaltwelle auf den Spulenabschnitten AM bzw. ME herrschen, sowie durch die Ströme $\left(J_{10}\right)_{p}$ und $\left(J_{30}\right)_{p}$, die zu derselben Zeit in den beiden Spulenhälften fließen.

Betrachten wir diese Werte als gegeben, dann ist uns auch die in die Spule laufende Spannungswelle $V_{1 \mathbf{p}}^{\prime}$ bekannt. Denn da der Spulenanfang zwangsweise auf dem konstanten Potential $\mathrm{E}$ gehalten wird, so ist für die p-te Schaltwelle, wie für alle vorhergehenden Wellen:

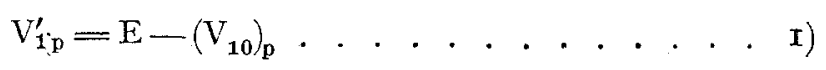


Auch die übrigen Wellen sind nicht unabhängig voneinander. Die Stetigkeit in $M$ verlangt nämlich (Abb. 6):

sowie

$$
\left[\mathrm{V}_{10}+\mathrm{V}_{2}^{\prime \prime}\right]_{\mathrm{p}}=\mathrm{V}_{\mathrm{2p}}=\left[\mathrm{V}_{30}+\mathrm{V}_{\mathrm{2}}^{\prime}\right]_{\mathrm{p}} \text {. . . . . . . . . . 2) }
$$

$$
\left[\mathrm{J}_{10}+\mathrm{J}_{2}{ }^{\prime \prime}\right]_{\mathrm{p}}=\mathrm{J}_{2 \mathrm{p}}=\left[\mathrm{J}_{30}+\mathrm{J}_{2}{ }^{\prime}\right]_{\mathrm{p}} \text {. . . . . . . . . 3) }
$$

Analog setzen wir für die Spannungen und Ströme am Spulenende:

sowie

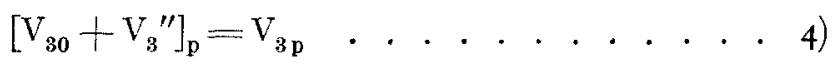

$$
\left[\mathrm{J}_{30}+\mathrm{J}_{3}{ }^{\prime \prime}\right]_{\mathrm{p}}=\mathrm{J}_{3 \mathrm{p}} \text {. . . . . . . . . . . 5) }
$$

wobei $J_{\mathbf{s} p}$ den Momentanwert des in die Leitung abfließenden Stromes bedeutet. Da ferner diese Leitung durch den elektromagnetischen Ausgleich-Vorgang innerhalb der Spule nicht induziert werden soll, besteht zwischen Spannung und Strom der einfache Zusammenhang:

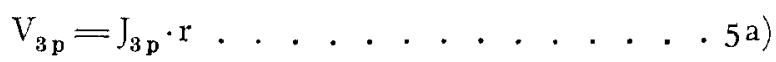

Es ist daher für den Vorgang in der Spule gleichgüitig; ob an ihrem Ende eine lange Leitung vom Wellenwiderstande $r$ oder ein gleichgroßer induktionsfreier Widerstand zur Erde abgezweigt wird.

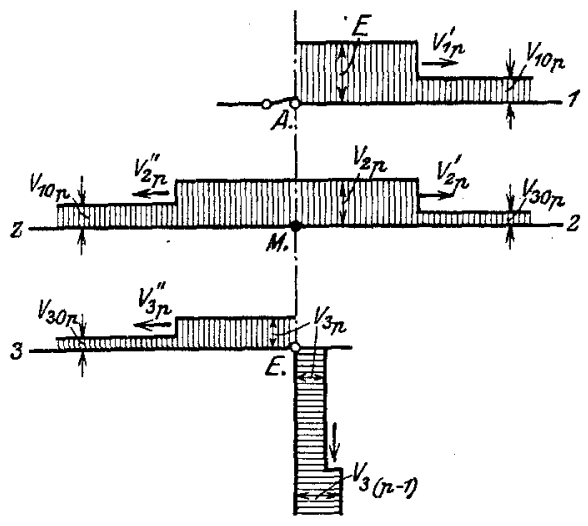

Abb. 6a. Spannungsverteilung.

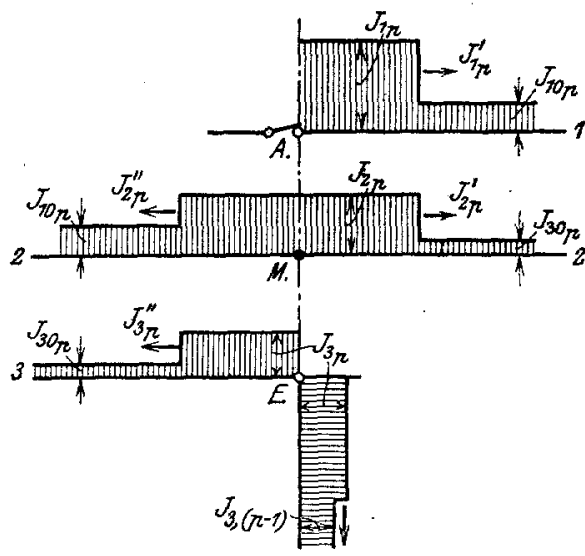

Abb. 6b. Stromverteilung.

Abb. 6a u. b. Zur Formulierung der Wanderwellengleichungen für parallele Windungsabschnitte.

Die Anfangs- und Grenzbedingungen des Problems haben wir mit den Gesetzen der elektromagnetischen Wechselwirkung zwischen den parallelen Spulenabschnitten zu kombinieren. - Bei zwei magnetisch gekoppelten Stromkreisen mit konzentrierten Induktivitäten pflegt man die totale Feldspannung eines Kreises in eine EMK der Selbstinduktion und eine EMK der Wechselinduktion aufzulösen. Geradeso ist es auch bei der Behandlung von Wanderwellen vorteilhaft ${ }^{1}$ ) die Amplitude der Spannungswelle aus einer dem eigenen Strom $\left(J_{1}\right)$ entsprechenden Komponente

$$
\mathrm{V}_{11}=\mathrm{J}_{1} \mathrm{w}
$$

und einer von der benachbarten Leitung induzierten Komponente

abzuleiten. Dabei bezeichnet

$$
\mathrm{V}_{21}=\mathrm{J}_{2} \mathrm{x}
$$

$$
\mathrm{w}=\mathrm{c} \cdot \mathrm{L}_{1}
$$

$(c=$ Wanderwellengeschwindigkeit) den Eigen-Wellenwiderstand der aus Leitung I

1) Vgl. die zitierte Arbeit von Wagner. 
und Erde gebildeten Schleife, deren Selbstinduktion pro Längeneinheit $L_{1}$ ist. Ana$\log$ bedeutet

$$
\mathrm{x}=\mathrm{c} \cdot \mathrm{M}
$$

den Wechsel-Wellenwiderstand zwischen den aus Leitung I bzw. 2 mit Erde als Rückleitung gebildeten Schleifen ( $\mathrm{M}=$ =Koeffizient der magnetischen Wechselinduktion pro Längeneinheit zwischen beiden Schleifen). Wir schreiben daher für irgendeine rechtsläufige Welle $V_{1_{p}}^{\prime}$ auf Leitung $I$ :

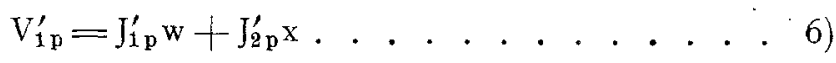

Dieselbe Überlegung liefert für den parallelen Windungsschnitt 2 :

$$
\mathrm{V}_{2 \mathrm{p}}^{\prime}=\mathrm{J}_{2 \mathrm{p}}^{\prime} \mathrm{w}+\mathrm{J}_{\mathrm{i} \mathrm{p}}^{\prime} \text {. . . . . . . . . . . . 7) }
$$

Analoge Gleichungen drücken die Wechselwirkung zwischen den Windungsabschnitten 2 und 3 aus. Da es sich aber hierbei um linksläufige Wellen handelt, haben wir das negative Vorzeichen zu gebrauchen, also:

bzw.

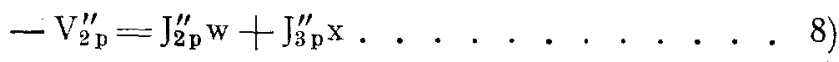

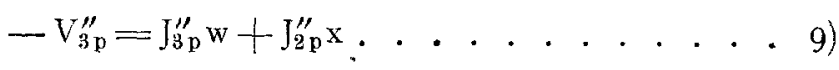

Diese Gleichungen im Verein mit den Anfangs - und Grenzbedingungen I bis 5 genügen, um für irgendeinen Umlauf $\mathrm{p}$ sämtliche Unbekannte zu berechnen. Ich will mich jedoch hierbei nicht aufhalten, sondern sogleich die Lösung mitteilen. Zur Abkürzung der Formeln gebrauchen wir den Blondelschen Koeffizienten der Gesamtstreuung zwischen den aus zwei parallelen Windungsabschnitten mit Erde als Rückleitung gebildeten Schleifen:

$$
\sigma=\mathrm{I}-\frac{\mathrm{M}^{2}}{\mathrm{~L}^{2}}=\mathrm{I}-\frac{\mathrm{x}^{2}}{\mathrm{~W}^{2}}
$$

Ferner eine Hilfsgröße von der Dimension eines Wellenwiderstandes

$$
\mathrm{z}=\mathrm{r}(\mathrm{I}+\sigma)+2 \mathrm{w} \sigma \text {. }
$$

Wir erhalten dann zunächst zwei Beziehungen zwischen den Wellen $V_{2 p}^{\prime \prime}$ und $V_{3 p}^{\prime \prime}$, nämlich:

und

$$
\left.V_{2 p}^{\prime \prime} \frac{2 w}{x}-V_{3 p}^{\prime \prime}=\left[V_{1}^{\prime}+\frac{w}{x}\left(V_{30}-V_{10}\right)+\frac{w^{2} \sigma}{x}\left(J_{10}-J_{30}\right)\right]_{p} . . . I 2\right)
$$

$$
V_{2 p}^{\prime \prime} \frac{x r}{w}-V_{3 p}^{\prime \prime}\left(\frac{r}{w}+\sigma\right)=-\left[J_{30} r-V_{30}^{\prime} \cdot \sigma \ldots . . . . . . \quad I_{3}\right)
$$

Daraus folgt für die Spannungswellen des p-ten Umlaufes

$$
\begin{aligned}
& \mathrm{V}_{3 \mathrm{p}}^{\prime \prime}=\left[\mathrm{V}_{1}^{\prime}+\frac{\mathrm{w}}{\mathrm{x}}\left(\mathrm{V}_{30}-\mathrm{V}_{10}\right)+\frac{\mathrm{w}^{2} \sigma}{\mathrm{x}}\left(\mathrm{J}_{10}-\mathrm{J}_{30}\right)\right] \frac{\mathrm{r}(\mathrm{I}-\sigma)}{\mathrm{z}}+\left[\mathrm{J}_{30} \mathrm{r}-\mathrm{V}_{30}\right]_{\mathrm{p}} \frac{2 \mathrm{w} \sigma}{\mathrm{z}} \\
& \left.\mathrm{V}_{2 \mathrm{p}}^{\prime \prime}=\left[\mathrm{V}_{1}^{\prime}+\frac{\mathrm{w}}{\mathrm{x}}\left(\mathrm{V}_{30}-\mathrm{V}_{10}\right)+\frac{\mathrm{w}^{2} \sigma}{\mathrm{x}}\left(\mathrm{J}_{10}-\mathrm{J}_{30}\right)\right]_{\mathrm{p}} \frac{\mathrm{x}}{\mathrm{z}}\left(\frac{\mathrm{r}}{\mathrm{w}}+\sigma\right)+\left[\mathrm{J}_{30} \mathrm{r}-\mathrm{V}_{30}\right]_{\mathrm{p}} \frac{\mathrm{x} \sigma}{\mathrm{z}} \mathrm{I}_{5}\right) \\
& \mathrm{V}_{2 \mathrm{p}}^{\prime}=\mathrm{V}_{2 \mathrm{p}}^{\prime \prime}+\left(\mathrm{V}_{10}-\mathrm{V}_{30}\right)_{\mathrm{p}} \text {. . . . . . . . . . . . . . . I6) }
\end{aligned}
$$

Sind diese Werte berechnet, so erhält man auch die Knotenpunktsspannungen $V_{\mathbf{2} p}$ und $V_{3 p}$ aus den Gl. 2) und 4).

Analog ergeben sich für die Stromwellen zunächst zwei Gleichungen zwischen $\mathrm{J}_{2 \mathrm{p}}^{\prime \prime}$ und $\mathrm{J}_{3 \mathrm{p}}^{\prime \prime}$ nämlich:

und

$$
\left.\mathrm{J}_{2 \mathrm{p}}^{\prime \prime} \mathrm{w}(\mathrm{I}+\sigma)+\mathrm{J}_{3 \mathrm{p}}^{\prime \prime} \mathrm{x}=-\left[\mathrm{V}_{1}{ }^{\prime}+\frac{\mathrm{w}}{\mathrm{x}}\left(\mathrm{V}_{30}-\mathrm{V}_{10}\right)+\frac{\mathrm{w}^{2} \sigma}{\mathrm{x}}\left(\mathrm{J}_{10}-\mathrm{J}_{30}\right)\right]_{\mathrm{p}} \frac{\mathrm{x}}{\mathrm{w}} \cdot . \quad \mathrm{I} 7\right)
$$

$$
\left.\mathrm{J}_{2 \mathrm{p}}^{\prime \prime} \mathrm{x}+\mathrm{J}_{3 \mathrm{p}}^{\prime \prime}(\mathrm{r}+\mathrm{w})=-\left[\mathrm{J}_{30} \mathrm{r}-\mathrm{V}_{30}\right]_{\mathrm{p}} \cdot \ldots . . . . \mathrm{I} 8\right)
$$


Hieraus folgt dann für die Stromwellen selbst:

$$
\begin{aligned}
& \mathrm{J}_{3 \mathrm{p}}^{\prime \prime}=\left[\mathrm{V}_{1}^{\prime}+\frac{\mathrm{w}}{\mathrm{x}}\left(\mathrm{V}_{30}-\mathrm{V}_{10}\right)+\frac{\mathrm{w}^{2} \sigma}{\mathrm{x}}\left(\mathrm{J}_{10}-\mathrm{J}_{30}\right)\right]_{\mathrm{p}} \frac{\mathrm{I}-\sigma}{\mathrm{z}}-\left[\mathrm{J}_{30} \mathrm{r}-\mathrm{V}_{30}\right]_{\mathrm{p}} \frac{\mathrm{I}+\sigma}{\mathrm{z}} \cdot \text { I9) } \\
& \left.J_{2 p}^{\prime \prime}=-\left[V_{1}^{\prime}+\frac{w}{x}\left(V_{30}-V_{10}\right)+\frac{w^{2} \sigma}{x}\left(J_{10}-J_{30}\right)\right]_{p} \frac{x}{w z}\left(I+\frac{r}{w}\right)+\left[J_{30} r-V_{30}\right]_{p} \frac{x}{w Z}-20\right)
\end{aligned}
$$

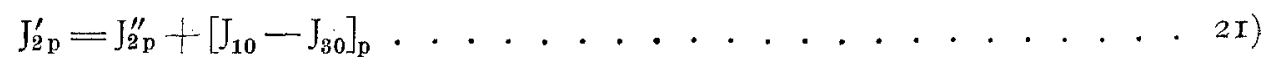

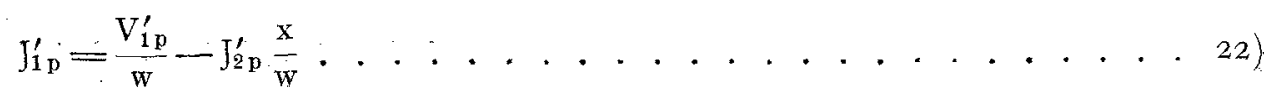

Endlich berechnen wir noch den gesamten in die Spule eintretenden Strom:

$$
\mathrm{J}_{\mathbf{1}}^{\prime}=\left[\mathrm{J}_{10}+\mathrm{J}_{1}^{\prime}\right]_{\mathrm{p}} \text {. . . . . . . . . . . . . 23) }
$$

und den am Spulenende austretenden Strom

$$
J_{3 p}=\left[J_{30}+J_{3}{ }^{\prime \prime}\right]_{p}=\frac{V_{3 p}}{r}
$$

Damit sind sämtliche Unbekannte ermittelt.

\section{Die Berechnung der Strom- und Spannungswellen während der beiden ersten Intervalle $(p=I$ und $p=I)$.}

\section{Das erste Schwingungsintervall (Index $\mathrm{p}=\mathrm{I}$ ).}

Bevor die Spannung $\mathrm{E}$ auf die Spule geschaltet wurde, waren die Spule und die angeschlossene Leitung spannungs- und stromlos. Für die ersten Schaltwellen sind daher die Anfangsbedingungen:

$$
\left.\begin{array}{rl}
\left(\mathrm{V}_{10}\right)_{\mathrm{I}} & =\left(\mathrm{V}_{30}\right)_{\mathrm{I}}=0 \\
\left(\mathrm{~J}_{10}\right)_{\mathrm{I}} & =\left(\mathrm{J}_{30}\right)_{\mathrm{I}}=0 \\
V_{1 \mathrm{I}}^{\prime} & =\mathrm{E}
\end{array}\right\}
$$

Für die übrigen Spannungswellen entnehmen wir den Gl, I4) und I5) sofort die Lösung:

$$
\begin{array}{r}
\left.V_{3 \mathrm{I}}=\mathrm{V}_{3 \mathrm{I}}^{\prime \prime}=\mathrm{E} \cdot \frac{\mathrm{r}}{\mathrm{z}}(\mathrm{I}-\sigma) \ldots . . . . .25\right) \\
\left.\mathrm{V}_{2 \mathrm{I}}=\mathrm{V}_{2 \mathrm{I}}^{\prime}=\mathrm{V}_{2 \mathrm{I}}^{\prime \prime}=\mathrm{E} \frac{\mathrm{x}}{\mathrm{z}}\left(\frac{\mathrm{r}}{\mathrm{w}}+\sigma\right) . . . . .26\right)
\end{array}
$$

Ferner betragen die Stromwellen nach G]. (9), 20) und 22):

$$
\begin{aligned}
& \mathrm{J}_{3 \mathrm{I}}=\mathrm{J}_{3 \mathrm{I}}^{\prime \prime}=\frac{\mathrm{E}}{\mathrm{z}}(\mathrm{I}-\sigma) \quad \ldots . \cdots \cdot \ldots .27 \\
& \left.\mathrm{~J}_{2 \mathrm{I}}=\mathrm{J}_{2 \mathrm{I}}^{\prime}=\mathrm{J}_{\mathrm{I}}^{\prime \prime}=-\frac{\mathrm{Ex}}{\mathrm{z}} \frac{\mathrm{w}}{\mathrm{w}}\left(\mathrm{I}+\frac{\mathrm{r}}{\mathrm{w}}\right) \ldots . . . \cdot 28\right) \\
& J_{1 \mathrm{I}}=J_{1 \mathrm{I}}^{\prime}=\frac{\mathrm{E}}{\mathrm{z}}\left(\mathrm{I}+2 \frac{\mathrm{r}}{\mathrm{w}}+\sigma\right) \ldots . . . .
\end{aligned}
$$

Die Bedeutung der Hilfsgröße z erkennen wir am besten aus Gl. 27). Ihr zufolge ist der erste Ladestromstoß, der in die angeschlossene Leitung entsandt wird, ebenso groß, als träfe sie die volle Spannung $\mathrm{E}$ bei einem Wellenwiderstand

$$
\left.\frac{\mathrm{z}}{\mathrm{I}-\sigma}=\mathrm{r}\left(\frac{\mathrm{I}+\sigma}{\mathrm{I}-\sigma}+2 \frac{\mathrm{w}}{\mathrm{r}} \frac{\sigma}{\mathrm{I}-\sigma}\right) . . . . \mathrm{II} \mathrm{I}\right)
$$

Je fester hiernach die elektrische und magnetische Koppelung der Windungen, d. h. 
je geringer ihre Streuung $\sigma$, um so mehr nähert sich $\frac{Z}{I-\sigma}$ dem wahren Wellenwiderstande $r$ der Leitung, um so größer wird daher der Ladestromsto $B J_{3 I}$ und die Ladespannung $\mathrm{V}_{3 \mathrm{I}}$. Im Grenzfalle $\sigma=0$ dringt die volle Einschaltspannung in die Leitung ein. Setzen wir dageg̀en beispielsweise

so wird

$$
\sigma=0,2 \quad \text { und } \quad \mathrm{r}=\mathrm{w} \text {; }
$$

$$
\stackrel{z}{\sim}-\bar{\sigma}=2 r \text {, }
$$

d. h. Ladestrom und Ladespannung sind nur halb so groß, als würde die Leitung ohne Vorschaltung der Spule an gleiche Spannung gelegt. Für diese Verhältnisse sind die Abb. 3 bis 5 entworfen. Die Zahlenwerte sind:

$$
\begin{array}{ll}
\mathrm{V}_{1 \mathrm{I}}^{\prime}=\mathrm{E} & \mathrm{J}_{1 \mathrm{I}}=2 \frac{\mathrm{E}}{\mathrm{W}}, \\
\mathrm{V}_{2 \mathrm{I}}=0,67 \mathrm{E} & \mathrm{J}_{2 \mathrm{I}}=-\mathrm{I}, \mathrm{I} 2 \frac{\mathrm{E}}{\mathrm{W}}, \\
\mathrm{V}_{3 \mathrm{I}}=0,5 \mathrm{E} & \mathrm{J}_{3 \mathrm{I}}=0,5 \frac{\mathrm{E}}{\mathrm{w}} .
\end{array}
$$

Das zweite Schwingungsintervall (Index $\mathrm{p}=\mathrm{II}$ ).

Nachdem die Spannungs- und Stromwellen eine Windung durchlaufen haben, exreichen sie die Knotenpunkte A, M und E. Der damit hergestellte Ladezustand ist der Anfangszustand, dem sich die an den Knotenpunkten reflektierten Wellen überlagern. Für dieses zweite Intervall ist daher gemäß $A b b .3 c, d$ und $4 a, b$

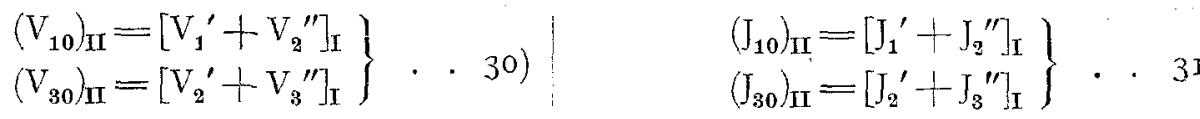

Die am Spulenanfang eindringende Entladewelle $V_{1 \text { II }}^{\prime}$ entsteht durch totale Reflektion der von innen auftreffenden Ladewelle $V_{21}^{\prime \prime}$. Es gilt daher:

$$
\mathrm{V}_{1 \mathrm{II}}^{\prime}=-\mathrm{V}_{2 \mathrm{I}}^{\prime \prime} \text {. . . . . . . . . . . 32) }
$$

Treten wir mit diesen Anfangsbedingungen in die allgemeinen Gl. I4) bis I6) und I9) bis 23) ein, so erhalten wir fïr die Amplituden der Wanderwellen: .

$$
\left.\left.\begin{array}{l}
V_{3 \mathrm{II}}^{\prime \prime}=-\left[V_{2}+V_{3} \frac{4 \times \sigma}{z}\right]_{I} \\
V_{2 I I}^{\prime \prime}=-\left[V_{3}+V_{2} \frac{4 \times \sigma}{z}\right]_{I}
\end{array}\right\} \quad 33\right)\left\{\begin{array}{l}
J_{2 I I}^{\prime \prime}=E-\left[2 V_{3}+V_{2} \frac{4 \times \sigma}{z}\right]_{I}^{\prime \prime} \\
\left.J_{2 I}^{\prime \prime}=-\left[J_{3}+J_{2} \frac{4 \times \sigma}{z}\right]_{I} \frac{4 \times \sigma}{z}\right]_{I} \\
J_{2 I I}^{\prime}=J_{1 I}-\left[2 J_{3}+J_{2} \frac{4 \times \sigma}{2}\right]_{I} \\
J_{1 \mathrm{II}}^{\prime}=\left[-3 J_{1}+2 J_{2} \frac{4 \times \sigma}{z}+5 J_{3}\right]_{I} \frac{x}{2 \mathrm{~W}}
\end{array}\right\}
$$

Daraus berechnen sich sodann auch die Spannungen und Ströme an den Knotenpunkten A, M, E:

$$
\left.\begin{array}{l}
V_{1 I I}=E \\
V_{2 I I}=E+\left[V_{2}\left(I-\frac{4 \times \sigma}{z}\right)-V_{3}\right]_{I} \\
V_{3 I I}=V_{3 I}\left(I-\frac{4 \times \sigma}{z}\right)
\end{array}\right\}
$$

$$
\left.\left.\begin{array}{l}
J_{1 \mathrm{II}}=\left[\mathrm{J}_{1}+\mathrm{J}_{2}\right]_{\mathrm{I}}+\mathrm{J}_{1 \mathrm{II}}^{\prime} \\
\mathrm{J}_{2 \mathrm{II}}=\mathrm{J}_{1 \mathrm{I}}+\left[\mathrm{J}_{2}\left(\mathrm{I}-\frac{4 \times \sigma}{\mathrm{z}}\right)-\mathrm{J}_{3}\right]_{\mathrm{I}} \\
\mathrm{J}_{3 \mathrm{II}}=\mathrm{J}_{3 \mathrm{I}}\left(\mathrm{I}-\frac{4 \times \sigma}{\mathrm{z}}\right)
\end{array}\right\} 36\right)
$$


Unser Beispiel liefert gemäß den Anfangsbèdingungen

$$
\begin{aligned}
& \left(V_{10}\right)_{I I}=I, 67 \mathrm{E} \\
& \left(V_{30}\right)_{I I}=I, I 7 E .
\end{aligned}
$$

$$
\begin{aligned}
& \left(\mathrm{J}_{10}\right)_{\mathrm{II}}=0,88 \frac{\mathrm{E}}{\mathrm{W}} \\
& \left(\mathrm{J}_{30}\right)_{\mathrm{II}}=-0,62 \frac{\mathrm{E}}{\mathrm{W}}
\end{aligned}
$$

für die Schaltwellen:

$$
\begin{aligned}
& V_{1 \mathrm{II}}^{\prime}=-0,67 \mathrm{E} \\
& V_{2 \mathrm{II}}^{\prime}=-0,3 \mathrm{E} \\
& V_{2 \mathrm{II}}^{\prime \prime}=-0,8 \mathrm{E} \\
& V_{3 \mathrm{II}}^{\prime \prime}=-0,893 \mathrm{E}
\end{aligned}
$$

$$
\begin{aligned}
& J_{1 \mathrm{II}}^{\prime}=-2,0 \mathrm{x} \frac{\mathrm{E}}{\mathrm{w}} \\
& \mathrm{J}_{2 \mathrm{II}}^{\prime}=\mathrm{I}, 5 \frac{\mathrm{E}}{\mathrm{W}} \\
& \mathrm{J}_{2 \mathrm{II}}^{\prime \prime}=0 \\
& J_{3 \mathrm{II}}^{\prime \prime}=0,893 \frac{\mathrm{E}}{\mathrm{W}}
\end{aligned}
$$

und für die elektromagnetischen Größen in der Knotenpurkten

$$
\begin{aligned}
& V_{1 \mathrm{II}}=\mathrm{E} \\
& \mathrm{V}_{2 \mathrm{XI}}=0,87 \mathrm{E} \\
& V_{3 \mathrm{II}}=0,277 \mathrm{E}
\end{aligned}
$$

$$
\begin{aligned}
& \mathrm{J}_{1 \mathrm{II}}=-\mathrm{I}, \mathrm{I} 3 \frac{\mathrm{E}}{\mathrm{w}} \\
& \mathrm{J}_{2 \mathrm{II}}=0,88 \frac{\mathrm{E}}{\mathrm{W}} \\
& \mathrm{J}_{3 \mathrm{II}}=0,277 \frac{\mathrm{E}}{\mathrm{w}}
\end{aligned}
$$

Bei dem das, zweite Intervall ausfüllenden Entladevorgange steigt also die Knotenpunktspannung in $M$ wieder nahezu auf den vollen Wert der Schaltspannung E: Dagegen sinkt die Spannung am Spulenende nahezu auf die Hälfte des Spannungsstoßes $\left(V_{3 I}\right)$, welcher im Schaltmoment in die Leitung eindrang.

\section{Die Differenzengleichung der Spannungswellen.}

Die Formeln $\mathrm{I}_{4}$ bis 23 gestatten bereits eine sukzessive Berechnung der Stromund Spannungswellen aufeinanderfolgender Schwingungsintervalle. Sie lassen jedoch ohne eine wiederholte Durchführung dieser zeitraubenden Rechnung nicht erkennen, wie sich der Schwingungsvorgang im großen und ganzen abwickeln wird. Wir wären
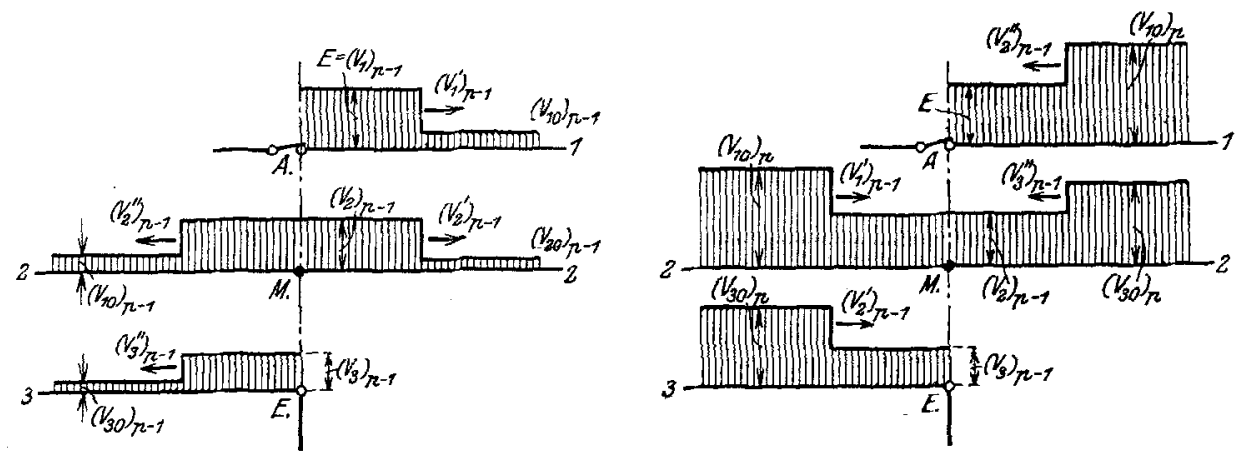

Abb. 7. Zum Vergleich der Spannungsverteilung $V_{10}$ und $V_{30}$ am Anfang des (p-r)-ten und p-ten Intervalles.

ein großes Stück weiter, wenn wir die Amplitude irgendeiner Spannungswelle allein durch die Einschaltespannung $\mathbf{E}$ und die Ordnungszahl $\mathrm{p}$ des Schwingungsintervalles ausdrücken könnten. Es wird sich zeigen, daß dies in der Tat möglich ist.

Um hierhin zu gelangen, müssen wir eine Beziehung suchen, die während einiger 
aufeinanderfolgender Zeitintervalle die Amplituden ein und derselben Spannungswelle verknüpft. Eine Gleichung dieser Art

$$
f\left(V_{p}, V_{p-1}, V_{p-2}, \ldots, V_{p-n}\right)=0
$$

nennt man eine Differenzengleichung $n$-ten Grades für die variable $V$.

Wir beginnen damit, daß wir in den Gl. I2) und I3) die Größen des Anfangszustandes $\left(\left(\mathrm{V}_{10}\right)_{\mathrm{p}},\left(\mathrm{V}_{\mathbf{3 0}}\right)_{\mathrm{p}},\left(\mathrm{J}_{10}\right)_{\mathrm{p}}\right.$ und $\left.\left(\mathrm{J}_{30}\right)_{\mathrm{p}}\right)$ durch die Spannungswellen des vorhergehenden Schwingungsintervalls (Index $\mathrm{p}-\mathrm{I}$ ) ausdrücken. Zum Teil ist diese Umformung aus Abb. 7 direkt abzulesen. Vergleicht man nämlich den Anfangszustand der p-ten und $(\mathrm{p}-\mathrm{I})$-ten Welle, so ergibt sich:

$$
\left.\begin{array}{l}
\left(\mathrm{V}_{10}\right)_{\mathrm{p}}=\left[\mathrm{V}_{10}+\mathrm{V}_{1}{ }^{\prime}+\mathrm{V}_{2}^{\prime \prime}\right]_{\mathrm{p}-1} \\
\left(\mathrm{~V}_{30}\right)_{\mathrm{p}}=\left[\mathrm{V}_{30}+\mathrm{V}_{2}{ }^{\prime}+\mathrm{V}_{3}^{\prime \prime}\right]_{\mathrm{p}-1}
\end{array}\right\} . . . . . . .
$$

Da in Gl. Iz) nur die Differenz der Anfangspotentiale auftritt, so bilden wir

$$
\left[\mathrm{V}_{30}-\mathrm{V}_{10}\right]_{\mathrm{p}}=\left[\left(\mathrm{V}_{30}-\mathrm{V}_{10}\right)-\left(\mathrm{V}_{2}^{\prime \prime}-\mathrm{V}_{2}^{\prime}\right)\right]_{\mathrm{p}-1}+\left[\mathrm{V}_{3}^{\prime \prime}-\mathrm{V}_{1}^{\prime}\right]_{\mathrm{p}-1}
$$

oder da sich nach Gl. 2) die Glieder unter der ersten eckigen Klammer auf der rechten Seite fortheben:

$$
\left.\left[V_{30}-V_{10}\right]_{p}=\left[V_{3}^{\prime \prime}-V_{1}^{\prime}\right]_{p-1} \cdot . . . . . . .38\right)
$$

Auf demselben Wege ergibt sich durch die Differenz der Anfangsströme:

$$
\left[\mathrm{J}_{10}-\mathrm{J}_{30}\right]_{\mathrm{p}}=\left[\mathrm{J}_{1}{ }^{\prime}-\mathrm{J}_{3}{ }^{\prime \prime}\right]_{\mathrm{p}-1} \text {. . . . . . . . . . . 39) }
$$

Hierin haben wir aber noch die Stromwellen der rechten Seite durch die Spannungswellen des gleichen Intervalles auszudrücken. Aus den Gl. 6) bis 9) entwickeln wir zunächst

$$
\left[\mathrm{J}_{1}{ }^{\prime}-\mathrm{J}_{3}{ }^{\prime \prime}\right]_{\mathrm{p}-1}=\frac{\mathrm{I}}{\mathrm{w}^{2} \sigma}\left[\mathrm{V}_{1}{ }^{\prime} \mathrm{w}-2 \mathrm{~V}_{2}{ }^{\prime \prime} \mathrm{x}+\mathrm{V}_{3}{ }^{\prime \prime} \mathrm{w}\right]_{\mathrm{p}-1}+\left[\mathrm{V}_{30}-\mathrm{V}_{10}\right]_{\mathrm{p}-1}
$$

und mit Benutzung von Gl. 38):

$$
\left.\left[J_{10}-\mathrm{J}_{30}\right]_{\mathrm{p}}=\frac{\mathrm{I}}{\mathrm{W}^{2} \sigma}\left[\mathrm{V}_{1}^{\prime}-2 \mathrm{~V}_{\mathrm{a}}^{\prime \prime} \mathrm{x}+\mathrm{V}_{\mathrm{3}}^{\prime \prime} \mathrm{w}\right]_{\mathrm{p}-1}+\left[\mathrm{V}_{\mathrm{3}}^{\prime \prime}-\mathrm{V}_{1}^{\prime}\right]_{\mathrm{p}-2} \ldots . .40\right)
$$

Beachtet man noch das Reflexionsgesetz für den Spulenanfang:

$$
V_{2(p-1)}^{\prime \prime}=-V_{1 p}^{\prime}
$$

so liefert die rechte Seite der Gl. I2)

$$
\left[\mathrm{V}_{1}^{\prime}+\frac{\mathrm{W}}{\mathrm{x}}\left(\mathrm{V}_{30} \cdot-\mathrm{V}_{10}\right)+\frac{\mathrm{w}^{2} \sigma}{\mathrm{x}}\left(\mathrm{J}_{10}-\mathrm{J}_{30}\right)\right]_{\mathrm{p}}=3 \mathrm{~V}_{1 \mathrm{p}}^{\prime}+\frac{2 \mathrm{w}}{\mathrm{x}} \mathrm{V}_{3(\mathrm{p}-1)}^{\prime \prime}+\left[\mathrm{V}_{3}^{\prime \prime}-\mathrm{V}_{1}\right]_{\mathrm{p}-2}
$$

In ähnlicher Weise entwickeln wir für die rechte Seite der Gl. I3):

$$
-\left[J_{30} r-V_{30}\right]_{p} \sigma=\left(\frac{r}{W}-\sigma\right) V_{1 p}^{\prime}+\frac{R x}{w^{2}} V_{1(p-1)}^{\prime}+\left(\frac{r}{w}-\sigma\right)\left[V_{3}^{\prime \prime}-V_{1}^{\prime}\right]_{p-2}
$$

Diese Werte führen wir in die GI. I2) und 13 ) ein und erhalten

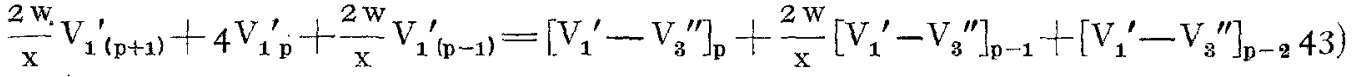

$$
\begin{aligned}
& \left.\frac{r x}{w} V_{1(p+1)}^{\prime}+2 r V_{1 p}^{\prime}+\frac{r x}{w} V_{1(p-1)}^{\prime}=(r+w o)\left[V_{1}^{\prime}-V_{a}^{\prime \prime}\right]_{p}+(r-w \sigma)\left[V_{1}^{\prime}-V_{3}^{\prime \prime}\right]_{p-2} 44\right)
\end{aligned}
$$

Damit haben wir zwei Differenzengleichungen zweiten Grades für die zwei Unbekannten $V_{1}^{\prime}$ und $V_{3}^{\prime \prime}$ gewonnen. Doch treten noch beide Spannungen in beiden Gleichungen auf. Wir müssen daher eine der Unbekannten eliminieren, wobei sich der Grad der Differenzengleichung erhöht. 
Zunächst leiten wir durch einfache Umformung aus 43) und 44) zwei neue Gleichungen $a b$, die zur weiteren Behandlung besser geeignet sind:

$$
\begin{aligned}
& z \cdot V_{1(p+1)}^{\prime}+\frac{2 x}{w}(r+2 w \sigma) V_{1}^{\prime}+z V_{1(p-1)}^{\prime}=2(\mathrm{r}+\mathrm{w} \sigma)\left[V_{1}^{\prime}-V_{3}^{\prime \prime}\right]_{p-1}+2 x \sigma\left[V_{1}^{\prime}-V_{3}^{\prime \prime}\right]_{p-2} \\
& (z-4 \mathrm{w} \sigma) \mathrm{V}_{1(\mathrm{p}+1)}^{\prime}+\frac{2 \mathrm{x}}{\mathrm{w}}(\mathrm{r}-2 \mathrm{w} \sigma) \mathrm{V}_{1 \mathrm{p}}^{\prime}+(\mathrm{z}-4 \mathrm{w} \sigma) \mathrm{V}_{1(\mathrm{p}-1)}^{\prime} \\
& =-2 \times \sigma\left[\mathrm{V}_{1}^{\prime}-\mathrm{V}_{3}{ }^{\prime \prime}\right]_{\mathrm{p}}+2(\mathrm{r}-\mathrm{w} \sigma)\left[\mathrm{V}_{1}^{\prime}-\mathrm{V}_{3}{ }^{\prime \prime}\right]_{\mathrm{p}-1} \text {. . . . . } 4
\end{aligned}
$$

Dazu fügen wir die beiden folgenden Gleichungen, die aus den vorigen entsteherr, in dem wir den Index der Spannungswellen um I erhöhen bzw. erniedrigen:

$$
\begin{gathered}
z \cdot V_{1(p+2)}^{\prime}+\frac{2 \mathrm{x}}{\mathrm{w}}(\mathrm{r}+2 \mathrm{w} \sigma) V_{1}^{\prime}(\mathrm{p}+1)+z V_{1 \mathrm{p}}^{\prime}=2(\mathrm{r}+\mathrm{w} \sigma)\left[\mathrm{V}_{1}^{\prime}-\mathrm{V}_{3}^{\prime \prime}\right]_{\mathrm{p}}+2 \mathrm{x} \sigma\left[\mathrm{V}_{1}^{\prime}-\mathrm{V}_{3}^{\prime \prime}\right]_{\mathrm{p}-1} \\
45 \mathrm{a}) \\
(z-4 \mathrm{w} \sigma) \mathrm{V}_{1 \mathrm{p}}^{\prime}+\frac{2 \mathrm{x}}{\mathrm{w}}(\mathrm{r}-2 \mathrm{w} \sigma) \mathrm{V}_{1(\mathrm{p}-1)}^{\prime}+(\mathrm{z}-4 \mathrm{w} \sigma) \mathrm{V}_{1(\mathrm{p}-\mathrm{z})} \\
\left.=-2 \times \sigma\left[V_{1}^{\prime}-\mathrm{V}_{3}^{\prime \prime}\right]_{\mathrm{p}-1}+2(\mathrm{r}-\mathrm{w} \sigma)\left[\mathrm{V}_{1}^{\prime}-\mathrm{V}_{3}^{\prime \prime}\right]_{\mathrm{p}-2} . . . .46 \mathrm{a}\right)
\end{gathered}
$$

Endlich bilden wir:

$$
-(45)(\mathrm{r}-\mathrm{w} \sigma)+(46) \cdot(\mathrm{r}+\mathrm{w} \sigma)+(45 \mathrm{a}+46 \mathrm{a}) \mathrm{x} \sigma
$$

Dann fallen alle Glieder auf der rechten Seite fort und es bleibt

$V_{1(p+2)}^{\prime}+\frac{4 \times \sigma}{z} V_{1(p+1)}^{\prime}-\frac{2 r(I-\sigma)}{z} V_{1 p}^{\prime}-\frac{4 \times \sigma}{z} V_{1(p-1)}^{\prime}+\left(I-\frac{4 W \sigma}{z}\right) V_{1(p-2)}^{\prime}=0$

Dies ist die gesuchte Differenzgleichung für die Amplitude der Spannungswelle, die vom Schalter aus in die Spule eindringt. Gleichlautende Differenzengleichungen liefern auch die übrigen Wanderwellen $V_{3}^{\prime \prime}$, ferner

und

$$
\left.V_{2 p}^{\prime \prime}=-V_{1(p+1)}^{\prime} \cdot . . . . . . . .32 a\right)
$$

$$
V_{2 p}^{\prime}=-V_{1(p+1)}^{\prime}+\left[V_{1}^{\prime}-V_{3}^{\prime \prime}\right]_{p-1}
$$

Die Gleichungen der Anfangsspannungen $V_{10}, V_{\mathbf{3 0}}$ und der Knotenpunktsspannungen $V_{2}, V_{3}$ unterscheiden sich nur durch ein Zusatzglied $\frac{\mathrm{E}}{\frac{4 \mathrm{r} \sigma}{\mathrm{z}}}$ auf der ręchten Seite. $Z$. B.: $\mathrm{V}_{3(p+2)}+\frac{4 \mathrm{x} \sigma}{\mathrm{z}} \mathrm{V}_{3(\mathrm{p}+1}-\frac{2 \mathrm{r}(\mathrm{I}-\sigma)}{\mathrm{z}} \mathrm{V}_{3 \mathrm{p}}-\frac{4 \mathrm{x} \sigma}{\mathrm{z}} \mathrm{V}_{3(\mathrm{p}-1)}+\left(\mathrm{I}-\frac{4 \mathrm{~W} \sigma}{\mathrm{z}}\right) \mathrm{V}_{3(\mathrm{p}-2)}=\frac{4 \mathrm{r} \sigma}{\mathrm{z}} \mathrm{E}$

Wir können jedoch auch die Knotenpunktsspannungen durch die Wanderwellen-Amplituden ausdrücken, indem wir entwickeln

und

$$
V_{2 p}=\left(V_{10}\right)_{p}+V_{2 p}^{\prime \prime}=E-V_{1 p}^{\prime}+V_{2 p}^{\prime \prime} \quad \text {. . . . . . }
$$

oder

$$
\mathrm{V}_{3 \mathrm{p}}=\left(\mathrm{V}_{30}\right)_{\mathrm{p}}+\mathrm{V}_{3 \mathrm{p}}^{\prime \prime}=\left[\mathrm{V}_{30}-\mathrm{V}_{10}\right]_{\mathrm{p}}+\mathrm{E}+\left[\mathrm{V}_{3}^{\prime \prime}-\mathrm{V}_{1}^{\prime}\right]_{\mathrm{p}}
$$

$$
\mathrm{V}_{3 \mathfrak{p}}=\left[\mathrm{V}_{3}^{\prime \prime}-\mathrm{V}_{1}^{\prime}\right]_{\mathfrak{p}}+\left[\mathrm{V}_{3}^{\prime \prime}-\mathrm{V}_{1}^{\prime}\right]_{\mathfrak{p}-1}
$$

Gl. 47) ist eine Differenzengleichung vierten Grades mit konstanten Koeffizienten. Man kann sie lösen, wenn man ihre "charakteristische Gleichung."

$$
\left.\mathrm{y}^{4}+\frac{4 \mathrm{x} \sigma}{\mathrm{z}} \mathrm{y}^{3}-\frac{2 \mathrm{r}(\mathrm{I}-\sigma)}{\mathrm{z}} \mathrm{y}^{2}-\frac{4 \mathrm{x} \sigma}{\mathrm{z}} \mathrm{y}+\left(\mathrm{I}-\frac{4 \mathrm{w} \sigma}{\mathrm{z}}\right)=0 . . . \quad . \quad 50\right)
$$

zu lösen vermag. Besitzt diese Gleichung vier reelle Wurzeln $\mathrm{y}_{1}, \mathrm{y}_{2}, \mathrm{y}_{3}, \mathrm{y}_{4}$, so lautet die Lösung der Differenzengleichung:

$$
V_{1 p}^{\prime}=C_{1} y_{1}^{p}+C_{2} y_{2}^{p}+C_{3} y_{3}^{p}+C_{4} y_{4}^{p}
$$


wobei die willkürlichen Konstanten $\mathrm{C}_{1}$ bis $\mathrm{C}_{4}$ aus den Anfangsbedingungen der Aufgabe zu berechnen sind.

Sind dagegen die Wurzeln der charakteristischen Gleichung konjugiert-komplex, etwa:

$$
\left.\left.\begin{array}{l}
\mathrm{y}_{12}=\mathrm{u}(\cos \varphi+\mathrm{i} \sin \varphi \\
\mathrm{y}_{34}=\mathrm{v}(\cos \psi \pm \mathrm{i} \sin \psi
\end{array}\right\} \quad . . . . . . . .52\right)
$$

so lautet die Lösung der Differenzengleichung:

$$
\left.\begin{array}{rl}
V_{1}^{\prime} & =\mathrm{u}^{\mathrm{p}}\left(\mathrm{C}_{1} \cos \mathrm{p} \varphi+\mathrm{C}_{2} \sin \mathrm{p} \varphi\right)+\mathrm{v}^{\mathrm{p}}\left(\mathrm{C}_{3} \cos \mathrm{p} \psi+\mathrm{C}_{4} \sin \mathrm{p} \psi\right) \\
& =\mathrm{A} \cdot \mathrm{u}^{\mathrm{p}} \cos (\mathrm{p} \varphi-\alpha)+\mathrm{B} \cdot \mathrm{v}^{\mathrm{p}} \cos (\mathrm{p} \psi-\beta)
\end{array}\right\} .
$$

Glücklicherweise ist G1. 50) für nicht zu große Werte der Streuung in einfacher Weise und doch mit guter Annäherung lösbar. Ist nämlich $\sigma^{2}$ klein gegen $I$, so kann man an Stelle des mittleren Gliedes das nur wenig geänderte Glied

$$
\frac{2 \mathrm{r}\left(\mathrm{I}-\sigma-2 \sigma^{2}\right)}{\mathrm{z}} \mathrm{y}^{2} \approx \frac{2 \mathrm{r}(\mathrm{I}-\sigma)}{\mathrm{z}} \mathrm{y}^{2} \text {. . . . . . . . 54) }
$$

einführen. In diesem Falle läßt sich die Gleichung als das Produkt zweier Ausdrücke vom zweiten Grade darstellen:

$$
\left(\mathrm{y}^{2}-2 \frac{\mathrm{x}}{\mathrm{w}} \frac{\mathrm{r}(\mathrm{I}+\sigma)}{\mathrm{z}} \mathrm{y}+\mathrm{I}-\frac{4 \mathrm{w} \sigma}{\mathrm{z}}\right) \cdot\left(\mathrm{y}^{2}+2 \frac{\mathrm{x}}{\mathrm{w}}+\mathrm{I}\right)=0 \ldots . .
$$

$\mathrm{Da}$ beide Klammerwerte für sich verschwinden müssen, so sind die ersten beiden Wurzeln der charakteristischen Gleichung.

$$
\mathrm{y}_{12}=\frac{\mathrm{x}}{\mathrm{w}} \cdot \frac{\mathrm{r}(\mathrm{I}+\sigma)}{\mathrm{z}} \pm \mathrm{i} \sqrt{\sigma} \cdot \frac{\sqrt{\mathrm{r}^{2}(\mathrm{I}+\sigma)^{2}-4 \mathrm{w}^{2} \sigma}}{\mathrm{z}} \ldots \ldots .
$$

oder in der oben gegebenen Schreibweise

$$
\begin{aligned}
& u=\sqrt{\mathrm{I}-\frac{4 \mathrm{w} \sigma}{\mathrm{z}}} \text {........ . . 56a) } \\
& \left.\cos \varphi=\frac{\mathrm{x}}{\mathrm{w}} \frac{\mathrm{r}(\mathrm{x}+\sigma)}{\mathrm{zu}} . . . . . . . .56 \mathrm{~b}\right)
\end{aligned}
$$

und

$$
\left.\sin \varphi=\sqrt{\sigma} \frac{\sqrt{\mathrm{r}^{2}(\mathrm{I}+\sigma)^{2}-4 \mathrm{w}^{2} \sigma}}{\mathrm{zu}} \text {....... . . . } 56 \mathrm{c}\right)
$$

Dagegen liefert die dritte und vierte Wurzel:

$$
\mathrm{y}_{34}=-\frac{\mathrm{x}}{\mathrm{w}} \pm \mathrm{i} \sqrt{\sigma} . . . . . . . . . .
$$

oder in trigonometrischer Form

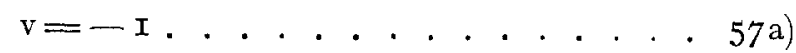

$$
\begin{aligned}
& \cos \psi=\frac{\mathrm{x}}{\mathrm{w}}=\sqrt{\mathrm{I}-\sigma} \text {. . . . . . . . . . . 57b) } \\
& \sin \psi=\sqrt{\sigma} \text {. . . . . . . . . . . . 57 c) }
\end{aligned}
$$

Betrachten wir zunächst nur die erste Hälfte der Lösung, d. h. die Wurzeln $y_{12}$ und die ihnen entsprechende Wanderwellenserie

Da

$$
\left.\left(\mathrm{V}_{1 \mathrm{p}}^{\prime}\right)_{12}=\mathrm{A} \cdot \mathrm{u}^{\mathrm{p}} \cdot \cos (\mathrm{p} \varphi-\alpha) . . . . . . . .53 \mathrm{a}\right)
$$

$$
\mathrm{u}=\sqrt{\mathrm{I}-\frac{4 \mathrm{w} \sigma}{\mathrm{z}}}
$$


eine positive Größe und stets kleiner als $I$ ist, so bedeutet die Funktion

$$
\mathrm{u}^{\mathrm{p}}=\mathrm{f}(\mathrm{p})
$$

einen treppenförmig abfallenden Linienzug, der einer erIöschenden Exponentialfunktion

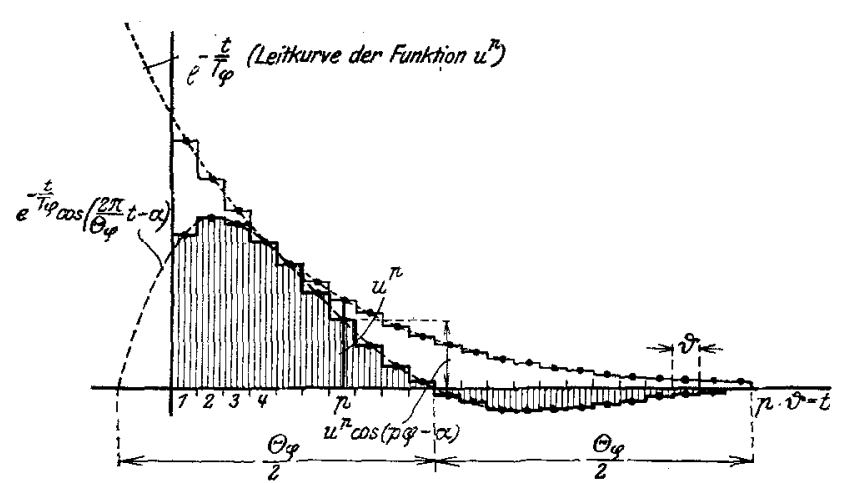

Abb. 8. Charakter der Funktion $\mathfrak{u}^{\mathrm{p}}$ und $\mathfrak{u}^{\mathrm{p}} \cos (\mathrm{p} \varphi-\alpha)$.

bzw.

$$
\mathrm{e}^{-\frac{\vartheta}{\mathrm{T}_{\varphi}}}=\sqrt{\mathrm{I}-\frac{4 \mathrm{~W} \sigma}{z}}
$$

$$
\mathrm{e}^{-\frac{\mathrm{t}}{\mathrm{T}_{\varphi}}}
$$

gleicht (Abb. 8). Ist die Streuung klein, so läßt sich auch die äquivalente Zeitkonstante $T_{\varphi}$ leicht angeben: Einem Schwingungsintervall entspricht die Zeit $\vartheta$, welche die Wanderwelle braucht, um eine Windung zu durchlaufen. Wir haben daher für den Vergleich

$$
\mathrm{t}=\mathrm{p} \cdot \theta
$$

einzuführen und erhalten

$$
\frac{\vartheta}{\mathrm{T}_{\varphi}}=-\frac{\mathrm{I}}{2} \ln \left(\mathrm{I}-\frac{4 \mathrm{w} \sigma}{\mathrm{z}}\right) \approx \frac{2 \mathrm{w} \sigma}{\mathrm{z}} .
$$

Die Zeitkonstante der Dämpfung beträgt somit:

$$
\left.\mathrm{T}_{\varphi}=-\frac{2 \vartheta}{\ln \left(\mathrm{I}-\frac{4 \mathrm{w} \sigma}{\mathrm{z}}\right)} \approx \vartheta_{0} \frac{2}{2 \mathrm{w} \sigma}=\vartheta\left(\mathrm{I}+\frac{\mathrm{r}}{2 \mathrm{w}} \frac{\mathrm{I}-\mathrm{T} \sigma}{\sigma}\right) \ldots . .5^{8}\right)
$$

Danach ist die Dämpfung der ersten Komponente der Wanderwellen um so geringer:

I. je größer die Windungslänge $(\vartheta !)$ d. h. die Selbstinduktion der Spule,

2. je geringez der Wellenwiderstand der Spule im Verhälnis zum Wellenwiderstand der angeschlossenen Leitung,

3. je geringer die Streuung zwischen den Spulenwindungen.

Bezeichnete der Faktor $\mathrm{u}^{\mathrm{p}}$ die Dämpfung der Wanderwellenschwingungen, so bestimmt der Faktor

ihre Periodendauer

$$
\cos (\mathrm{p} \varphi-\alpha)=\cos \left(\frac{\varphi}{\vartheta} \cdot \mathrm{t}-\alpha\right)
$$

$$
\left.\Theta_{\varphi}=\frac{2 \pi}{\varphi} \cdot \vartheta \quad \ldots . . . . . .59\right)
$$

Alles in allem ändert sich daher die Amplitude der ersten Komponente nach einem treppenförmigen Linienzug, dessen Leitkurve eine abklingende Sinusschwingung

ist (Abb. 8).

$$
\mathrm{e}^{-\frac{\mathrm{t}}{\mathrm{T}_{\varphi}} \cdot \cos \left(\frac{2 \pi}{\Theta_{\varphi}} \mathrm{t}-\alpha\right)}
$$

Wesentlich anders ist das Gesetz, welches der zweite Anteii der Lösung befolgt.

$$
\left.\left(\mathrm{V}_{1 \mathrm{p}}^{\prime}\right)_{34}=\mathrm{B} \cdot \mathrm{v}^{\mathrm{p}} \cos (\mathrm{p} \psi-\beta) . . . . . .53 \mathrm{~b}\right)
$$


Vor allem ist diese Schwingung weit schwächer gedämpft wie die vorige. Nach der oben mitgeteilten Näherungslösung wäre sie sogar vollkommen ungedämpft, da abgesehen vom Vorzeichen

$$
\left|\mathrm{v}^{\mathrm{p}}\right|=\left|\mathrm{I}^{\mathrm{p}}\right|=\mathrm{I}
$$

gefunden wurde. Eine genauere Rechnung lehrt jedoch, daß auch bei widerstandsloser Spule die Zeitkonstante $\mathrm{T}_{\psi}$ zwar groß, aber nicht unendlich wird, und daß sie für nicht zu kleine Werte von $r$ nach der Gleichung

$$
\mathrm{T}_{\psi}=\vartheta \cdot 2 \frac{\mathrm{r}}{\mathrm{w}} \cdot \frac{\mathrm{I}+\sigma}{\sigma^{3}} \ldots . . . . . .
$$

geschätzt werden kann. Wir werden später hierauf zurückkommen.

Sodann ist $\mathrm{v}$ negativ. Die Wanderwelle wechselt somit nach jedem Umlauf ihr Vorzeichen, soweit nicht die Multiplikation mit der cos-Funktion einen nochmaligen Vorzeichen-Wechsel bedingt.

Endlich ist ihre Periode

$$
\Theta_{\psi}=\frac{2 \pi}{\psi} \vartheta=\vartheta \cdot \frac{2 \pi}{\operatorname{arc~sin} \sqrt{\sigma}} \text {. . . . . . . . . }
$$

in erster Annäherung nur von der Windungslänge und der Streuung der Spule, nicht aber wie $\Theta_{\varphi}$ auch von dem Wellenwiderstand der angeschlossenen Leitung abhängig ${ }^{1}$ ). Erst wenn $\sigma$ sehr groß wird, versagt dieses Gesetz, denn dann muß die Substitution 54) $\mathrm{zu}$ Trugschlüssen führen.

Nach alledem ist der Charakter der zweiten Wanderwellen-Komponente durch einen rechteckigen Wellenzug nach Art von Abb. 9 wiederzugeben. Der Wechsel der Ordinaten erinnert an eine Schwebung. Diese Schwebung besteht noch, wenn die erste Schwingung $\left(\mathrm{V}_{\mathbf{1}}{ }^{\prime}\right)_{1,2}$ längst verklungen ist. Sie ist für die Spule allein, nicht für das Zusammenschalten der Spule mit anderen Leitungen charakteristisch und kann daher als Eigenschwingung der Spule angesprochen werden. In diesem Sinne würde der ersten Komponente nur die Aufgabe zufallen, den durch den Schaltprozeß eingeleiteten Ausgleichsvorgang allmählich in diese Eigenschwingung überzuführen. Wahrscheinlich wird sich diese Auffassung auch für die mehrdrähtige Spule als fruchtbar erweisen.

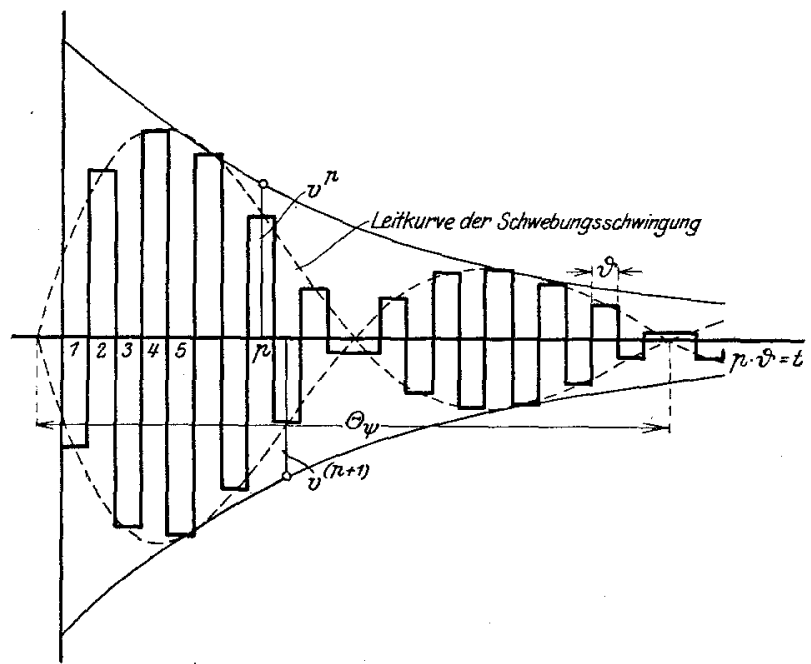

Abb. 9. Charakter der Funktion $\mathrm{v}^{\mathrm{p}} \cos (\mathrm{p} \psi-\beta)$.

Was für die Wanderzelle $V_{1}{ }^{\prime *}$ in Erfahrung gebracht wurde, gilt qualitativ auch für die übrigen Spannungswellen. Die Eigenschwingung der ganzen Spule stellt sich somit dar als das Spiel zweier gegenläufiger Wellen $\left(V_{1}^{\prime}\right.$ und $V_{2}^{\prime \prime}$ auf der ersten Windung, sowie $\mathrm{V}_{2}^{\prime}$ und $\mathrm{V}_{3}^{\prime \prime}$ auf der zweiten Windung), die zwischen den drei Knoten-

1) Für $r=0$ gilt die Lösung 57) sogar streng. Für $r=\infty$ wird:

was mit 57) immer noch gut übereinstimmt.

$$
\left.\mathbf{v}=-\mathrm{I} ; \quad \cos \psi=\sqrt{\frac{\mathrm{I}}{\mathrm{I}+\sigma}} ; \quad \sin \psi=\sqrt{\frac{\sigma}{\mathrm{I}+\sigma}}, \ldots . . .62\right)
$$


stellen $A, M, E$ unter stetigen Reflexionen hin und her laufen. Je kleiner hierbei der Wellenwiderstand der angeschlossenen Leitung und je loser die elektromagnetische Koppelung ist, welche die Wellen der beiden Windungen verknüpft, um so mehr wird sich die Eigenschwingung auch der angeschlossenen Leitung mitteilen und um so schneller wird sie daher erlöschen (vgl. 60).

Wenn ein merklicher Anteil der Eigenschwingung auch auf die Leitung hinter der Spule übertragen werden kann, so entsteht die Gefahr, daß die Spule für diesen Stromkreis als Schwingungserreger fungiert. Es ist daher von besonderem Interesse, zu untersuchen, welches Verhältnis zwischen den Eigenschwingungs-Amplituden in Spule und Leitung besteht.

Um hierüber Aufschluß zu exhalten, müssen wir zunächst die Konstanten der Eigenschwingung noch etwas genauer berechnen. An Stelle der Näherungslösung

$$
\mathrm{y}_{34}=(-\mathrm{I})^{\mathrm{p}}(\cos \psi+\mathrm{i} \sin \psi)
$$

versuchen wir jetzt den Ansatz:

$$
\mathrm{y}_{34}=(-\eta)^{\mathrm{p}} \cdot(\cos \psi \pm \mathrm{i} \sin \psi) \text {. . . . . . . . 63) }
$$

Treten wir damit in die charakteristische Gl. 50) ein, so ergeben sich für $\eta$ und $\psi$ zwei Bestimmungsgleichungen

und

$$
\left.\eta^{4} \cos ^{2} \psi-\eta^{3} \frac{2 \mathrm{x} \sigma}{\mathrm{z}} \cos \psi=\frac{\mathrm{I}}{4}\left[\eta^{4}+\eta^{2} \frac{2 \mathrm{r}(\mathrm{I}-\sigma)}{\mathrm{z}}+\mathrm{I}-\frac{4 \mathrm{w} \sigma}{\mathrm{z}}\right] \ldots 6_{4} \mathrm{a}\right)
$$

$$
\left.\cos \psi=\eta\left(\mathrm{r}+\eta^{2}\right) \cdot \frac{2 \times \sigma}{4 \mathrm{w} \sigma-\left(\mathrm{I}-\eta^{4}\right) z} \ldots . . . .64 \mathrm{~b}\right)
$$

Für $\eta=I$ würde aus der letzten Gleichung wie früher

hervorgehen. Setzen wir jetzt aber

$$
\cos \psi=\frac{\mathrm{x}}{\mathrm{w}}=\sqrt{\mathrm{I}-\sigma}
$$

$$
\eta=\mathrm{I}-\varepsilon \text {. . . . . . . . . . } 64 \mathrm{c} \text { ) }
$$

und betrachten $\varepsilon$ als kleine Größe, deren Potenzen gegen die Einheit zu vernachlässigen sind, so wird

$$
\cos \psi=\frac{\mathrm{x}}{\mathrm{w}}\left(\mathrm{I}+\frac{\mathrm{r}(\mathrm{I}-\sigma)}{\mathrm{w} \sigma}\right) \ldots . . . . .65
$$

und

oder bei nicht zu kleinen Werten von $r$ :

$$
\varepsilon=\frac{\mathrm{w}}{2 \mathrm{r}} \cdot \frac{\sigma^{3}}{\mathrm{I}+\sigma} \cdot \frac{\mathrm{I}}{\mathrm{I}+\frac{\sigma^{3}}{\mathrm{I}+\sigma} \cdot \frac{\mathrm{w}}{\mathrm{r}}\left(3+\frac{\mathrm{w}}{\mathrm{r}}\right)}
$$

$$
\varepsilon=\frac{\mathrm{w}}{2 \mathrm{r}} \cdot \frac{\sigma^{3}}{\mathrm{I}+\sigma} .
$$

Damit folgen aus Gl. 65a) die korrigierten Werte.

$$
\left.\begin{array}{l}
\cos \psi=\frac{\mathrm{x}}{\mathrm{W}}\left(\mathrm{I}+\frac{\sigma^{2}}{2}\right) \approx \frac{\mathrm{I}}{\sqrt{\mathrm{I}-\sigma}} \\
\sin \psi=\sqrt{\sigma-\frac{\sigma^{2}}{2}(\mathrm{I}+\sigma)} \approx \sqrt{\frac{\sigma}{\mathrm{I}+\sigma}}
\end{array}\right\} \ldots .
$$

Es empfiehlt sich auf Grund dieser Ergebnisse, auch die erste stark gedämpfte SchwingungsKomponente zu korrigieren. Wir werden sie in der Folge kurzerhand als "Anfangsschwingung" bezeichnen. Die verbesserte Gleichung ihrer Konstanten lautet: 


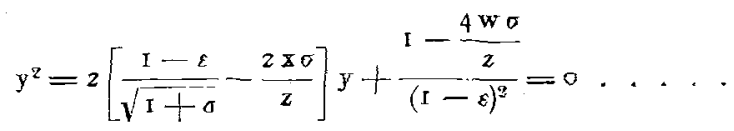

Bei nicht $z u$ grofer Streuung und $r \geqq w$ sind auch die Wurzeln dieser Gleichung konjugiert komplex. Es ist dann formell wieder die Lösung 52) zu verwenden, nur daf jetzt

und

erhalten wird.

$$
\left.\begin{array}{l}
u=(\mathrm{I}+\varepsilon) \sqrt{\mathrm{I}-\frac{4 \mathrm{w} \sigma}{z}} \\
\varphi=\arccos \left[\frac{\mathrm{s}-\varepsilon}{\sqrt{\mathrm{I}+\sigma}-\frac{2 \times \sigma}{z}}\right]
\end{array}\right\}
$$

Sind dagegen beide Wurzeln reell, so lautet die Lösung

$$
y_{12}=\left(\frac{1-\varepsilon}{\sqrt{I+\sigma}}-\frac{2 \times \sigma}{z}\right) \pm \sqrt{\left(\frac{I-\varepsilon}{\sqrt{I+\sigma}}-\frac{2 \times \sigma}{z}\right)^{2}-\frac{I-\frac{4 w \sigma}{z}}{(I-\varepsilon)^{2}} \ldots . . .}
$$

Besondere Erwähnung verdient ein ausgezeichneter Fall, der sich beim Anschluf eines Kabels an eine Spule praktisch verwirklichen läfat. Er liegt vor, wenn das letzte Glied in G1. 66) verschwindet, d. h. wenn das Verhältnis

besteht. Dann wird nämlich

$$
\frac{I}{W}==\frac{2 \sigma}{I+\sigma}
$$

und

oder wegen

$$
y_{1}=0
$$

einfacher:

$$
\mathrm{y}_{2}=2\left(\frac{\mathrm{I}-\varepsilon}{\sqrt{\mathrm{I}+\sigma}}=\frac{2 \times \sigma}{z}\right)
$$

$$
\varepsilon=\frac{\sigma^{2}}{4} \quad \text { und } \quad \frac{2 \times \sigma}{z}=\frac{\sqrt{1}-\sigma}{2}
$$

$$
\left.y_{8}=\frac{1}{\sqrt{1+\sigma}} \cdot . . . . . . . . . . .66 c\right)
$$

Die Gleichung der Anfangsschwingung lautet somit

$$
\left(\mathrm{V}_{\mathrm{p}}\right)_{12}=\mathrm{C} \cdot\left(\frac{\mathrm{I}}{\sqrt{\mathrm{I}+\sigma}}\right)^{\mathrm{p}} \text {. }
$$

Bei kleiner Streuung ist dies eine schwachgedämpfte Schwingung, die wie wir später sehen werden, für die Verwendung der Spule als Schutz-Drosselspule grofie Vorzüge besitzt.

Kehren wir nach dieser Abschweifung zü unserer ursprüngiichen Aufgabe zurück und versuchen an Hand der Formeln 65) die in die Leitung eindringende Welle zu kontrollieren. Wir brauchen hierfür nur die Spannung $V_{3}$ am Spulenende zu untersuchen. Denn das Zeitdiagramm dieser Spannung - nach Art der Diagramme 8 und 9 aufgezeichnet - gibt direkt die räumliche Wellenform auf der Leitung wieder. Man muß sich nur die Abszissen-Intervalle $\vartheta^{t}$ durch die Windungslänge l ersetzt denken. (Vgl. dazu Abb. 5.)

Nun beträgt die Spannung am Spulenende

$$
V_{3 p}=\left[V_{30}+V_{3}^{\prime \prime}\right]_{p}=\left[V_{30}-V_{10}\right]_{p}+\left[V_{3}^{\prime \prime}+V_{10}\right]_{p} \text {. . . . . }
$$

oder gemäß GI. I) und 38):

$$
\mathrm{V}_{3 p}=\mathrm{E}+\left[\mathrm{V}_{3}{ }^{\prime \prime}-\mathrm{V}_{1}^{\prime}\right]_{\mathrm{p}}+\left[\mathrm{V}_{3}^{\prime \prime}-\mathrm{V}_{1}^{\prime}\right]_{\mathrm{p}-1} \ldots \ldots 6_{7}
$$

Wir haben daher zuerst zu untersuchen, ob bzw. wie stark die Differenz

$$
\mathrm{D}_{\mathrm{p}}=\left[\mathrm{V}_{\mathrm{s}}{ }^{\prime \prime}-\mathrm{V}_{\mathrm{1}}\right]_{\mathrm{p}} \ldots \ldots \ldots
$$

die behandelte Eigenschwingung der Spule enthält.

Archiv E. Elektrotechnik. VIJ. 
$\mathrm{Zu}$ diesem Zwecke bedienen wir uns der Gl. 43), die ich zur besseren Übersicht noch einmal anschreibe:

$$
D_{p+1} \frac{x}{w}+2 D_{p}+D_{p-1} \frac{x}{w}=2 V_{2(p+1)}^{\prime \prime}+4 \frac{x}{w} V_{2 p}^{\prime \prime}+2 V_{2(p-1)}^{\prime \prime}
$$

Die Glieder auf der rechten Seite enthalten die Eigenschwingung jedenfalls stark. Wir setzen hierfür:

und versuchen den Ansatz

$$
\mathrm{V}_{2 \mathrm{p}}^{\prime \prime}=-\left(\mathrm{V}_{1}^{\prime}\right)_{\mathrm{p}+1}={\tilde{\mathrm{V}_{2}}}^{\prime \prime} \cdot(-\eta)^{\mathbf{p}} \cos (\mathrm{p} \psi-\gamma)
$$

$$
\mathrm{D}_{\mathrm{p}}=\tilde{\mathrm{D}} \cdot(-\eta)^{\mathrm{p}} \cos (\mathrm{p} \psi-\gamma)
$$

$\tilde{V}_{2}^{\prime \prime}$ und $\tilde{D}$ bedeuten somit die noch ungedämpften Amplituden der Schwebung, welche die Eigenschwingungen charakterisieren.

Unter diesen Annahmen liefert die linke Seite der Differenzengleichung 43):

$$
\tilde{\mathrm{D}} \cdot(-\eta)^{\mathrm{p}}\left\{\cos (\mathrm{p} \psi-\gamma)\left[2-\left(\eta+\frac{\mathrm{I}}{\eta}\right) \frac{\mathrm{x}}{\mathrm{w}} \cos \psi\right]+\sin (\mathrm{p} \psi-\gamma) \cdot\left(\eta-\frac{\mathrm{I}}{\eta}\right) \frac{\mathrm{x}}{\mathrm{w}} \sin \psi\right\}
$$

und wegen $\eta=\mathrm{r}-\varepsilon$ und Gl. $65 \mathrm{c}$ )

$$
2 \tilde{\mathrm{D}} \cdot(-\eta)^{\mathbf{p}} \cos (\mathrm{p} \psi-\gamma)\left[\sigma-(\mathbf{r}-\sigma) \frac{\sigma^{2}}{2}\right] .
$$

Die rechte Seite entwickeln wir analog und erhalten:

$$
\begin{aligned}
& 2 \tilde{V}_{a}^{\prime \prime} \cdot(-\eta)^{p}\left\{\cos (p \psi-\gamma)\left[2 \frac{\mathrm{x}}{\mathrm{w}}-\left(\eta+\frac{\mathrm{I}}{\eta}\right) \cos \psi\right]+\sin (\mathrm{p} \psi-\gamma) \cdot\left(\eta-\frac{\mathrm{I}}{\eta}\right) \sin \psi\right\} \\
& =-2 \tilde{\mathrm{V}}_{\mathrm{2}}^{\prime \prime}(-\eta)^{\mathrm{p}} \cos (\mathrm{p} \psi-\gamma) \cdot \frac{\mathrm{x}}{\mathrm{w}} \sigma^{2} .
\end{aligned}
$$

Unser Ansatz hat sich also bewährt, und zwar gilt für das Verhältnis der Schwebungen

$$
\frac{\tilde{D}}{\tilde{V}_{2}^{\prime \prime}}=-\frac{\sigma}{I-\frac{\sigma}{2}+\frac{\sigma^{2}}{2}} \cdot . . . \quad . \quad . \quad . \quad .69 \text { ) }
$$

Jetzt macht.auch die Bestimmung der Schwebungs-Amplitude $\tilde{V}_{3}$ in der Knotenpunktspannung $V_{\mathbf{3 p}}$ keine Schwierigkeiten mehr. Wir setzen:

Somit ergibt sich

$$
\begin{aligned}
& \mathrm{V}_{3 \mathrm{p}}=\mathrm{E}+\mathrm{D}_{\mathrm{p}}+\mathrm{D}_{\mathrm{p}-1} \text {. . . . . . . . . . . . . . . . 67) } \\
& \approx \mathrm{E}+\tilde{\mathrm{D}}(-\eta) \mathbf{p}[\cos (\mathrm{p} \psi-\gamma)-\cos ((\mathrm{p}+\mathrm{I}) \psi-\gamma)] \\
& =\mathrm{E}+\tilde{\mathrm{D}} \cdot 2 \sin \frac{\psi}{2}(-\eta)^{\mathbf{p}} \sin \left(\mathrm{p} \psi-\frac{\psi}{2}-\beta\right) \text {. }
\end{aligned}
$$

$$
\tilde{V}_{3}=\tilde{\mathrm{D}} \cdot 2 \sin \frac{\psi}{2}=(-) \tilde{\mathrm{V}}_{2}^{\prime \prime} \cdot 2 \sin \frac{\psi}{2}-\frac{\sigma}{\mathrm{I}-\frac{\sigma^{2}}{2}+\frac{\sigma^{2}}{2}} .
$$

Dies ist die anfängliche Amplitude der Schwebungen, welche die Eigenschwin. gung der Spule in die angeschlossene Leitung entsendet. Sie ist klcin für kleine Werte der Streuung $\sigma$, für welche man die abgekürzte Formel

$$
\tilde{V}_{3}=\tilde{V}_{2}^{\prime \prime} \sqrt{\frac{\sigma^{3}}{I+\sigma^{3}}}
$$

gebrauchen kann. So würde man für $\sigma=0,2$ nur

$$
\tilde{V}_{3}=0,09 \tilde{V}_{2}^{\prime \prime}
$$


zu gewärtigen haben. Dagegen ergäbe $\sigma=0,6$

$$
\tilde{\mathrm{V}}_{3}=0,47 \tilde{\mathrm{V}}_{2}^{\prime \prime} \text {. }
$$

Mit anderen Worten: die Eigenschwingung würde nahezu in halber Stärke auf die angeschlossene Leitung übergehen. Das kann gefährlich werden, wenn diese Schwingung (bei $r>w$ ) auch noch schwach gedämpft ist.

\section{Zahlenbeispiele.}

Es bleibt noch übrig an einigen Beispielen zu zeigen, wie die erkannten und diskutierten Gesetze dem ganzen Ausgleichvorgang das Gepräge geben. Da hierfür der Wellenwiderstand der angeschlossenen Leitung von sekundärer Bedeutung ist, wird im folgenden

$$
r=\mathrm{w}
$$

gesetzt. Unter diesen Annahmen soll das Verhalten zweier Spulen mit verschiedener Streuung

verglichen werden.

$$
\sigma=0,2 \text { bzw. } \sigma=0,6
$$

Um einen voliständingen Überblick zu gewinnen, muß man entweder die vier Integrationskonstanten $C_{1}$ bis $C_{4}$ der Differenzengleichung 47) bestimmen, oder man muB durch wiederholte Anwendung dieser Differenzengleichung die Wellenamplituden für eine große Zahl aufeinanderfolgender Intervalle berechnen. Wenn man bei großer Streuung nicht sicher ist, ob man die charakteristische Gl. 50) genau genug gelöst hat, jst der zuerst genannte Weg der zuverlässigere. In jedem Falle muß man zuvor für die Spannungswellen $V_{1}^{\prime}$ oder $V_{2}^{\prime}$ usw., deren Verhalten man aufklären will, die Ampli. tuden während vier aufeinanderfolgender Intervalle bestimmt haben.

Für die beiden ersten Intervalle ( $\mathrm{p}=\mathrm{I}$ und II) habe ich die Gleichungen bereits mitgeteilt. Für das dritte und vierte Intervall lassen sich Gl. I4) und I5) benutzen. Besser wandelt man diese jedoch durch Einführung der Formeln 4I) und 42) in Differenzengleichungen um, was auf die Ausdrücke führt:

$$
\begin{aligned}
& \left.V_{3 p}^{\prime \prime}=-V_{2(p-1)}^{\prime \prime}\left(I-\frac{4 r \sigma}{z}\right)-\left[V_{1}{ }^{\prime}-V_{3}^{\prime \prime}\right]_{p-1} \frac{2 \times r}{w z}+\left[V_{1}{ }^{\prime}-V_{3}^{\prime \prime}\right]_{p-2}\left(I-\frac{4 w \sigma}{z}\right): 7 I\right) \\
& V_{2 p}^{\prime \prime}=V_{2(p-1)}^{\prime \prime} \frac{2 x}{w} \cdot \frac{r-2 w \sigma}{2}+V_{3(p-1)}^{\prime \prime}\left[V_{1}{ }^{\prime}-V_{3}^{\prime \prime}\right]_{p-1} \frac{r(I-\sigma)}{z}-\left[V_{1}{ }^{\prime}-V_{3}{ }^{\prime \prime}\right]_{p-z} \cdot \frac{2 \times \sigma}{z}
\end{aligned}
$$

Also z. B. für $\mathrm{p}=\mathrm{III}$.

$$
\begin{aligned}
& V_{3 I I}^{\prime \prime}=-V_{2 I I}^{\prime \prime}\left(I-\frac{4 r \sigma}{z}\right)-V_{3 I} \frac{8 w \sigma \cdot r(I-\sigma)}{z^{2}}+\left(E-V_{3 I}\right)\left(I-\frac{4 w \sigma}{z}\right) . \\
& V_{2 I I I}^{\prime \prime}=V_{2 I I}^{\prime \prime} \frac{2 x}{w} \cdot \frac{x-2 w \sigma}{z}+V_{3 I I}^{\prime \prime}-V_{3 I} \frac{4 x \sigma \cdot r(I-\sigma)}{z^{2}}-\left(E-V_{3 I}\right) \frac{2 x \sigma}{z} .
\end{aligned}
$$

Unter der Annahme $\mathrm{r}=\mathrm{w}$ lassen sich diese Formeln noch etwas vereinfachen und liefern dann:

$$
\begin{aligned}
& \left.\mathrm{V}_{3 \mathrm{H}}^{\prime \prime}=\mathrm{E} \cdot \frac{\mathrm{I}-\sigma}{\mathrm{I}+3 \sigma}\left[\mathrm{I}-4 \sigma\left(\frac{\mathrm{I}-\sigma}{\mathrm{I}+3 \sigma}\right)^{2}\right] \ldots . . . . . . . .73\right) \\
& \left.V_{2 \mathrm{III}}^{\prime \prime}=-\mathrm{V}_{1 \mathrm{IV}}^{\prime}=\mathrm{E} \frac{\mathrm{x}}{\mathrm{w}}\left[\mathrm{I}-\frac{\sigma}{\mathrm{I}+3 \sigma}-2 \sigma \frac{\mathrm{I}+\sigma}{\mathrm{I}+3 \sigma}-2 \sigma\left(\frac{\mathrm{I}-\sigma}{\mathrm{I}+3 \sigma}\right)^{3}\right] \cdot . \cdot 74\right) \\
& V_{3 \mathrm{IV}}^{\prime \prime}=-\mathrm{E} \frac{\mathrm{x}}{\mathrm{W}} \frac{\mathrm{I}-\sigma}{\mathrm{I}+3 \sigma}\left[\mathrm{I}-2 \sigma \frac{\mathrm{I}+\sigma}{\mathrm{I}+3 \sigma}+2 \sigma(3+\sigma) \frac{(\mathrm{I}-\sigma)^{2}}{(\mathrm{I}+3 \sigma)^{3}}\right] . .7
\end{aligned}
$$


Entweder setzt man jetzt

$$
\begin{aligned}
& \mathrm{V}_{\mathrm{I}}=\mathrm{u} \cdot\left[\mathrm{C}_{1} \cos \varphi+\mathrm{C}_{2} \sin \varphi\right]+\mathrm{v}\left[\mathrm{C}_{3} \cos \psi+\mathrm{C}_{4} \sin \psi\right] \\
& \mathrm{V}_{\mathrm{II}}=\mathrm{u}^{2}\left[\mathrm{C}_{1} \cos 2 \varphi+\mathrm{C}_{2} \sin 2 \varphi\right]+\mathrm{v}^{2}\left[\mathrm{C}_{3} \cos 2 \psi+\mathrm{C}_{4} \sin 2 \psi\right] \\
& \mathrm{V}_{\mathrm{III}}=\mathrm{u}^{3}\left[\mathrm{C}_{1} \cos 3 \varphi+\mathrm{C}_{2} \sin 3 \varphi\right]+\mathrm{v}^{3}\left[\mathrm{C}_{3} \cos 3 \psi+\mathrm{C}_{4} \sin 3 \psi\right] \\
& \mathrm{V}_{\mathrm{IV}}=\mathrm{u}^{4}\left[\mathrm{C}_{1} \cos 4 \varphi+\mathrm{C}_{2} \sin 4 \varphi\right]+\mathrm{v}^{4}\left[\mathrm{C}_{3} \cos 4 \psi+\mathrm{C}_{4} \sin 4 \psi\right]
\end{aligned}
$$

und bestimmt aus diesen vier linearen Gleichungen die vier unbekannten Integrationskonstanten $\mathrm{C}_{1}, \mathrm{C}_{2}, \mathrm{C}_{3}$ und $\mathrm{C}_{4}$.

Oder man folgert aus der Differenzengleichung 47)

$$
\mathrm{V}_{\mathrm{V}}=-\frac{4 \mathrm{x} \sigma}{\mathrm{z}} \mathrm{V}_{\mathrm{IV}}+\frac{2 \mathrm{r}(\mathrm{I}-\sigma)}{\mathrm{z}} \mathrm{V}_{\mathrm{III}}+\frac{4 \mathrm{x} \sigma}{\mathrm{z}} \mathrm{V}_{\mathrm{II}}-\left(\mathrm{I}-\frac{4 \mathrm{~W} \sigma}{\mathrm{z}}\right) \mathrm{V}_{\mathrm{I}}
$$

und fährt in gleicher Weise fort $V_{\text {VI }}, V_{\text {VII }}$ usw. zu berechnen. Für unser Zahlenbeispiel habe ich den letzten Weg eingeschlagen und dabei die Werte der Tabelle I und II erhalten. Ein Teil dieser Ergebnisse ist in dem Zeitdiagramm Io bis I6 aufgetragen. Es ist notwendig diese Diagramme einzeln durchzusprechen. Denn jedes zeigt den Ausgleichvorgang von einer besonderen Seite.

\section{a) Die Wanderwellen.}

Betrachten wir zuerst das Spiel der rechts- und linksläufigen Wanderwellen auf beiden Windungsabschnitten AM und ME (vgl. auch Abb. I). Die Rechteckswellen in Abb. Ioa) zeigen den Amplituden-Wechsel der vom Schalter in die Spule eindringenden Wanderwelle, und zwar für die Spule mit der verhältnismäßig kleinen Streuung $\sigma=0,2$. Jede Rechtecksbreite entspricht einem Schwingungsintervall $\vartheta$, so daß das Diagramm als Zeitdiagramm gelesen werden kann. Positive Ordinaten bedeuten Ladewellen, negative Ordinaten Entladewellen. Im allgemeinen folgt also auf eine Ladewelle stets eine Entladewelle, wie sich dies schon bei der Betrachtung der einleitenden Schwingungsvorgänge im ersten Abschnitt ergab.

Gleichzeitig mit diesem Vorzeichen-Wechsel tritt der Schwebungscharakter des Ausgleichsvorganges in aller Klarheit hervor. Wir haben erfahren, daß diese eigenartige Selbstregulierung der Spule als solcher eigentümlich ist. Wir sehen jetzt, daß gegenüber dieser fast ungedämpften Eigenschwingung die durch die Verkettung der Spule mit fremden Stromkreisen bedingte schnellgedämpfte „Anfangsschwingung“ nur wenig ausmacht. Der treppenförmige Linienzug, dessen Ordinaten schraffiert sind, stellt diese Komponente der Wanderwellen dar. Sie gleicht, wie die Theorie es verlangt, einer abklingenden Sinusschwingung; aber sie bleibt so niedrig, daß sich das Nuliniveau der Schwebung nur wenig verschiebt. Deshalb muß der Ausgleichsvorgang mit einem Schwebungsbauch beginnen, der wenig kleiner als die volle Einschaltespannung ist.

Ich habe in Abb. Ioa die Scheitelpunkte der Rechteckswellen der resultierenden Schwingung durch zwei Leitkurven verbunden. Es sind Sinuslinien entgegengesetzten Vorzeichens, aber gleicher Höhe, deren Null-Linie die Leitlinie der starkgedämpften Anfangsschwingung bildet. Diese Darstellungsweise ermöglicht es, in dieselbe Figur die rechtsläufigen Wanderwellen $\mathrm{V}_{2}^{\prime}$ des parallelen Windungsabschnittes $\mathrm{ME}$ aufzunehmen. Ihre Leitlinien sind strichliert. Die mittlere, niedrige Leitlinie entspricht der schnellverlöschenden Anfangsschwingung, die beiden äußeren Leitkurven der Schwebungs-Eigenschwingung. Da die elektromagnetische Verkettung zwischen beiden Windungsabschnitten bei $\sigma=0,2$ noch ziemlich gut ist, sind die Wellen $V_{1}^{\prime}$ und $V_{2}^{\prime}$ nicht nur in ihrer Höhe, sondern auch in ihrer Phase wenig voneinander verschieden. Daß beide Schwebungen genau synchron sind, braucht wohl nicht besonders betont zu werden. 

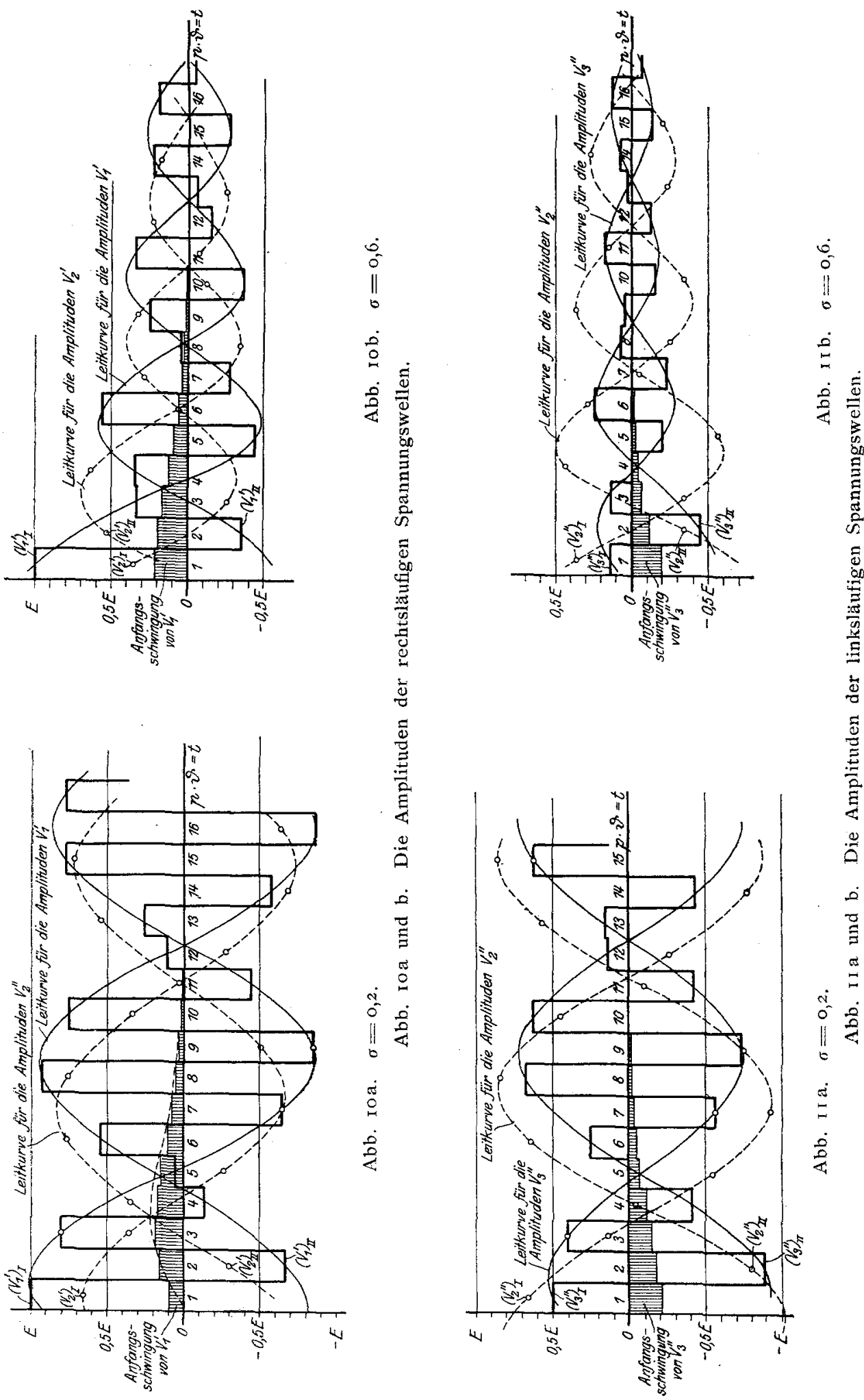
Wir wenden uns daher gleich zu Abb. Ira, die in derselben Darstellungsweise die linksläufigen Wellen $V_{2}^{\prime \prime}$ und $V_{3}^{\prime \prime}$ behandelt. Diesmal ist die Welle $V_{2 p}^{\prime \prime}=-V_{1(p+1)}^{\prime}$ die Primärwelle, von der die Induktionswirkungen ausgehen. Daher ist sie etwas größer als die induzierte Welle $V_{3}^{\prime \prime}$. Wiederum ist die Anfangsschwingung von sekundärer Bedeutung; da sie von Anbeginn klein und längst erloschen ist, während sich die Schwebungsschwingung noch so gut wie ungedämpft wiederholt.

Aus der Abb. Ioa die Zeitkonstante und Periodizität der Anfangsschwingung herzuleiten, ist schwierig. Man muß sich hier schon auf die Theorie verlassen. Dagegen ist für die Eigenschwingungs-Kamponente der Vergleich mit der Theorie leicht möglich: ihre Periode folgt mit Hilfe der Gleichung

$\mathrm{zu}$

$$
\psi=\arcsin \sqrt{\frac{\sigma}{I+\sigma}}=\arcsin 0,408=24^{\circ} \text {. . . . . . 65c) }
$$

$$
\Theta_{\psi}=\frac{360^{\circ}}{\psi} \cdot \vartheta=\mathrm{I} 5 \vartheta
$$

Es sollten also I5 Schwingungsintervalle in einer vollen Wellenlänge der Leitkurven enthalten sein. In der Tat entnehmen wir der Abbildung dasselbe Verhältnis. Ferner liefert die Theorie für die Dämpfungskonstante

$$
\mathrm{T}_{\psi}=2 \frac{\mathrm{x}+\sigma}{\sigma^{3}} \vartheta=3000 \vartheta . . . . . . .60
$$

Nach einer vollen. Wellenlänge der Leitkurve sollte daher die Amplitude nur im Verhältnis

$$
\mathrm{I}: \theta^{\frac{-\theta_{\psi}}{\mathrm{T}_{\psi}}}=\mathrm{I}: \mathrm{e}^{\frac{1}{200}}=\mathbf{I}: 0,995
$$

abgenommen haben. Auch diese verschwindende Dämpfung stimmt mit den Diagrammen überein.

Für die Anfangsschwingung liefert die Theorie das Gesetz

mit

$$
0,707^{\mathrm{p}} \cos (\mathrm{p} \varphi-c)
$$

$$
\varphi=\arccos \left[\frac{\frac{I}{\sqrt{I+\sigma}-\frac{2 \times \sigma}{z}}}{\sqrt{\mathrm{I}-\frac{4 \mathrm{w} \sigma}{z}}}\right]=\mathrm{r} 3^{0} \ldots . . . .
$$

Es sollten also $\frac{180^{\circ}}{13^{0}}=I_{4}$ Intervalle auf eine halbe Wellenlänge der Leitkurve gehen. Da aber für $\mathrm{p}=\mathrm{I} 4$ die Amplitude auf 0,8 Proz. ihres Anfangswertes gesunken ist, kann in den Diagrammen die Periodizität nicht in Erscheinung treten.

Wir haben noch zu untersuchen, wie die Lockerung der elektromagnetischen Koppelung den Charakter der Wanderwellenschwingung verändert. Diesem Zwecke dienen $\mathrm{Abb}$, Iob und $I I b$, die für eine Streuung $\sigma=0,6$ die eben besprochenen Diagramme wiederholen. Was bei dem Vergleich sofort ins Auge fällt, ist die Verkürzung der Schwebungsperiode und die ziemlich kräftige Dämpfung der Eigenschwingung. Beides war nach der Theorie zu erwarten. Annähernd sollte jetzt

und

$$
\left.\psi=\arcsin \sqrt{\frac{\sigma}{\mathrm{I}+\sigma}}=\arcsin 0,6 \mathrm{r} 2 \approx 38^{\circ} . . . . .65 \mathrm{c}\right)
$$

$$
\Theta_{\psi}=\frac{360^{\circ}}{\psi} \vartheta=9,5 \vartheta
$$


gefunden werden. Die Zeichnung liefert $\Theta_{y}=9,3 \vartheta$. Ferner haben wir eine Dämpfungskonstante

$$
\mathrm{T}_{\psi}=2 \frac{\mathrm{I}+\sigma}{\sigma^{3}} \cdot \vartheta=\mathrm{I}_{4}, 8 \vartheta \ldots . . . . . . .
$$

zu erwarten: Das Amplituden-Verhältnis zwischen zwei Punkten, die auf der Leitkurve eine volle Wellenlänge auseinanderliegen, sollte daher

$$
\mathrm{e}^{-\frac{\Theta_{\psi}}{\mathrm{T}_{\psi}}}=\mathrm{e}^{-0,642}=0,52
$$

betragen. Auch hier liefert die Zeichnung praktisch denselben Wert.

Es ist indessen nicht allein die Dämpfung, welche die Eigenschwingung der Spule gegenüber den früheren Diagrammen so stark geschwächt erscheinen läßt. Auch die Anfangswerte der ersten Intervalle liegen bereits tiefer. Das gilt insbesondere für die Amplituden $\mathrm{V}_{3}{ }^{\prime \prime}$, die infolge der losen elektromagnetischen Koppelung nur etwa halb so groß wie die erregenden Schwingungen $V_{2}^{\prime \prime}$ ausfallen. Schließlich sei noch auf die wachsende Phasenverschiebung zwischen den Leitkurven je zweier gleichläufiger Wellen hingewiesen. Trotz dieser Unterschiede erscheint noch immer die Eigenschwingung der Spule als das Charakteristikum des Ausgleichsvorganges, gegen welches die Anfangsschwingung völlig in den Hintergrund tritt.

\section{b) Die Knotenpunktsspannungen.}

Die Bedeutung der Anfangsschwingung liegt in dem Verhalten der Spannungen an den Punkten $M$ und $E$, die wir gewöhnlich als Knotenpunkte bezeichneten. Für sie bewirkt die Anfangsschwingung den Übergang von dem Anfangspotential $V_{\mathbf{I}}$ auf das Endpotential E, und wenn hier zu Anfang große Potentialdifferenzen bestanden, so kann die Anfangsschwingung die von der Eigenschwingung der Spule herrührende Komponente weit übertreffen.

Wie die Diagramme I2 und I3 zeigen, sind Spulenmitte und Spulenende keine echten Knotenpunkte in dem Sinne, daß sie von der Eigenschwingung der Spule überhaupt nicht in Mitleidenschaft gezogen würden. Doch ist diese Komponente schon in der Spannung $V_{2}$ der Spulenmitte $M$ ziemlich klein - die ankommende Welle $V_{1}{ }^{\prime}$ ist mehr als doppelt so groB - und in der Spannung $V_{3}$ am Spulenende ist sie bei niedriger Streuung kaum noch nennenswert.

Die Schnelligkeit, mit welcher die Anfangsschwingung am Spulenende abklingt, ist von großem praktischen Interesse. Denn, wie schon früher betont, ist das Zeitdiagramm für $V_{3}$ zugleich als Wegdiagramm für die Form der in die abgezweigte Leitung eindringenden Wanderwelle zu deuten. An die Stelle des Zeitintervalles $\vartheta$ tritt dann der Weg, den die Wanderwellen in derselben Zeit durchlaufen, d. i. die Windungslänge 1. Setzen wir also den Fall, die Spule solle für den dahinterliegenden Stromkreis als Überspannungsschutz fungieren, welcher die schroffe Stirn der von dem Schalter ausgehenden Spannungswellen zu brechen hat. Dann wird sie diesen Zweck offenbar um so besser erfüllen, je schwächer die Anfangsschwingung gedämpft ist.

Wenn man sich fragt, welchen Einfluß hierauf die Streuung hat, so zieht man am besten gleich die Abb. I3a und I3b zu Rate. Denselben Vergleich an Hand der entwickelten Theorie durchzuführen stößt nämlich insofern auf Schwierigkeiten, als für $\sigma=0,6$ die Anfangsschwingung nicht mehr periodisch, sondern aperiodisch gedämpft ist ${ }^{1}$ ). Im Einklang mit den Näherungsgleichungen 58) und 66c) beweisen die

1) Aus G1. 66 b) folgt nämlich:

$$
\begin{aligned}
& y_{1}=0,695 \\
& y_{2}=0,237 .
\end{aligned}
$$



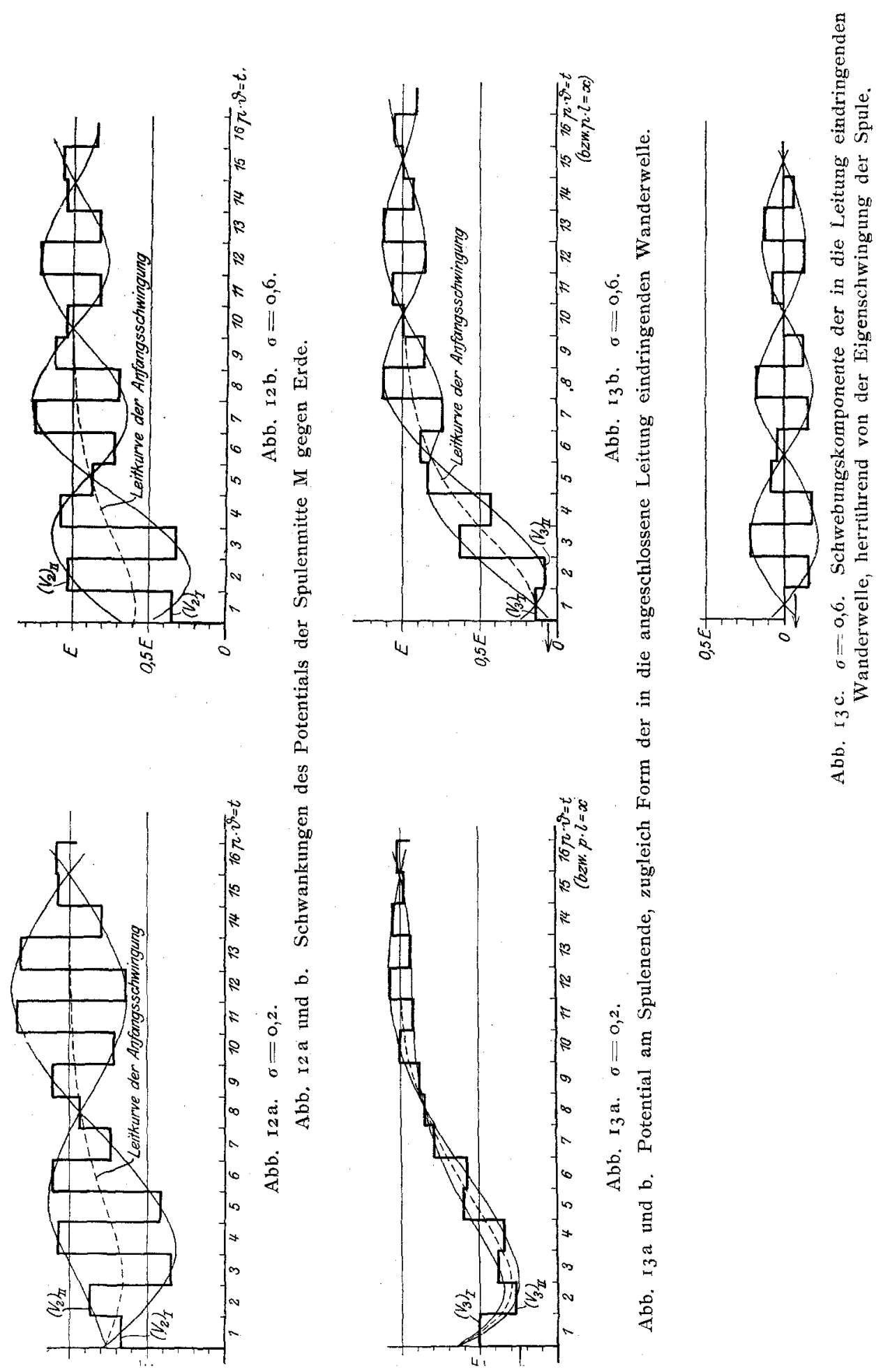
Diagramme, daß die Vergrößerung der Streuung die Dämpfung erhöht, also steile Wellenfronten begünstigt. Trotzdem wäre dies allein noch kein Grund, Schutzdrosselspulen mit geringer Streuung zu bevorzugen. Denn man braucht ja nur ihre Selbstinduktion - verkörpert durch die Windungslänge 1 - genügend zu vergrößern, um den Anstieg der Leitkurve der Anfangsschwingung beliebig zu mildern.

Was aber auch dann noch in Kauf genommen werden müßte, sind die hohen Stufen des treppenförmigen Anstieges, die von der starken Eigenschwingung innerhalb der Spulen herrühren. Bei der Spule mit großer Streuung habe ich diese Schwingungskomponente besonders herausgezeichnet, Abb. I3c, um ihren Schwebungscharakter deutlich hervortreten $\mathrm{zu}$ lassen. $\mathrm{Ob}$ sie auch als Schwingungserreger fur den angeschlossenen Stromkreis gefährlich werden könnte, vermag ich nicht zu beurteilen.

Trotzdem mit der Verringerung der Streuung die Amplitude der Eigenschwingung innerhalb der Spule wächst (vgl. Abb. Ioa und Iob), wird das Spulenende mehr und mehr zu einem echten Knotenpunkt, der diese Eigenschwingung nicht durchsickern läßt. Geringe Streuung wirkt also günstig. Aus Abb. I3a und $b$ entnehmen wir, daB schon bei $\sigma=0,2$ nur noch schwache Ausläufer in die Leitung eindringen, und wir konnten dieses Verhalten auch theoretisch begründen (Gl. 7oa). Dafür setzt aber die über die Leitung fortlaufende Wanderwelle sogleich mit einer steilen Wellenfront ${ }^{~} V_{3}$ von beträchtlicher Höhe ein. Man brauchte daher eigentlich noch eine zweite Vorschaltespule, um auch diese Wellenfront abzuschleifen.

Will man $V_{3 \mathrm{I}}$ trotz niedriger Streuung kleinhalten, so gibt es nur ein Mittel: Man muß den Wellenwiderstand $w$ der Spule möglichst groß gegen den Wellenwiderstand $r$ der Leitung machen ${ }^{1}$ ); denn wir fanden

$$
\mathrm{V}_{3 \mathrm{I}}=\mathrm{E} \cdot \frac{\mathrm{I}-\sigma}{\mathrm{I}+\sigma+\frac{\mathrm{w}}{\mathrm{r}} \cdot 2 \sigma} \quad \ldots . . . . . .25
$$

Beim AnschluB eines Kabels an eine Spule ist $r \leqslant w$ ohne weiteres erfüllt. In anderen Fällen dürfte dagegen die Einhaltung dieser Bedingung große Schwierigkeiten bereiten.

\section{c) Die Windungsspannung.}

Zu den bisher erörterten Gesichtspunkten tritt als letzter die Frage: Wie hoch wird die Spule selbst durch den Schaltvorgang beansprucht? Es verlohnt sich daher, den Wechsel des Potentials an einigen Punkten zu verfolgen.

Das Potential der Knotenpunkte wurde soeben untersucht. Die Differenz der Knotenpunktsspannungen gibt die Windungsspannung an gleicher Stelle. Die Windungsspannúng $\mathrm{E}-\mathrm{V}_{\mathrm{g}}$ am Spulenanfang kann den Diagrammen Iza und $\mathrm{b}$ entnommen werden. Sie ist am größten im dritten Intervall, wo sie bei beiden Spulen etwa $2 / 3$ der Schaltspannung $E$ beträgt. Höher liegt die Windungsspannung $V_{2}-V_{3}$ am Spulenende. Wie aus Tabelle I und II (Spalte Io) ersichtlich, erreicht sie hier bei $\sigma=0,23 / 4$ der Schaltspannung, bei $\sigma=0,6$ sogar nahezu die volle Schaltspannung. Es ergibt sich also das bemerkenswerte Resultat, daß die Endwindung höher beansprucht wird als die Anfangswindung.

1) $Z_{\mathfrak{u}}$ dem gleichen Ergebnis gelangt K. W. Wagner auf anderem Wege. Vgl. namentlich seinen Vortrag: Beanspruchurg und Schutzwirkung von Spulen bei schnellen Ausgleichsvorgängen. ETZ 1916 S. 266. 
Tabelle I. Die Spule mit geringer Streuung: $\sigma=0,2, \mathrm{r}=\mathrm{w}$.

(Die Schaltspannung $\mathrm{E}$ ist gleich I gesetzt.)

\begin{tabular}{|c|c|c|c|c|c|c|c|c|c|c|c|c|c|}
\hline \multirow{3}{*}{$\begin{array}{c}\text { Ordnungs- } \\
\text { zahl des } \\
\text { Intervalles } \\
\mathbf{p}\end{array}$} & \multicolumn{4}{|c|}{ Wanderwellen } & \multirow{2}{*}{\multicolumn{2}{|c|}{$\begin{array}{c}\text { Knoten- } \\
\text { punlkts- } \\
\text { potentiale }\end{array}$}} & \multirow{2}{*}{\multicolumn{2}{|c|}{$\begin{array}{l}\text { Lade- } \\
\text { zustand }\end{array}$}} & \multicolumn{5}{|c|}{ Windungsspannung } \\
\hline & \multicolumn{2}{|c|}{$\begin{array}{l}\text { Anfangs- } \\
\text { windung }\end{array}$} & \multicolumn{2}{|c|}{ Endwindung } & & & & & \multicolumn{2}{|c|}{ Vorderseite } & \multirow{2}{*}{$\left|\begin{array}{c|}\text { Rücken } \\
\mathrm{V}_{10} \\
-\mathrm{V}_{30}\end{array}\right|$} & \multicolumn{2}{|c|}{$\mid \begin{array}{c}\text { Rechte } \\
\text { Seite }\end{array}$} \\
\hline & $\mathrm{V}_{1}^{\prime}$ & $\mathrm{V}_{2}^{\prime \prime}$ & $\mathrm{V}_{2}^{\prime}$ & $\mathrm{V}_{3}^{\prime \prime}$ & $\mathrm{V}_{2}$ & $\mathrm{~V}_{8}$ & $\mathrm{~V}_{10}$ & $\mathrm{~V}_{30}$ & $E-V_{2}$ & $V_{2}-V_{3}$ & & $\left|\begin{array}{l}V_{10}-V_{30} \\
+V_{1}-V_{2}\end{array}\right|$ & $\begin{array}{r}V_{10}-V_{30} \\
+V_{2}^{\prime \prime}-V_{3}^{\prime \prime} \\
\end{array}$ \\
\hline $\mathrm{I}$ & I & 0,670 & 0,670 & 0,500 & 0,670 & 0,500 & 0 & $o$ & 0,330 & 0,170 & o & $0,33^{\circ}$ & 0,170 \\
\hline II & $-0,670$ & $-0,800$ & $|-0,300|$ & $-0,894$ & 0,870 & 0,277 & 1,670 & $I, 170$ & 0,130 & 0,593 & 0,500 & 0,130 & 0,594 \\
\hline III & 0,800 & 0,134 & $0,35^{8}$ & 0,400 & 0,334 & 0,377 & 0,200 & 0,024 & 0,666 & $-0,043$ & 0,224 & 0,666 & $-0,042$ \\
\hline IV & 0,134 & $-0,060$ & 0,340 & $-0,404$ & $\mathbf{I}, 074$ & 0,330 & 1,134 & 0,734 & $-0,074$ & 0,744 & 0,400 & $-0,074$ & 0,744 \\
\hline $\mathrm{V}$ & 0,060 & $-0,532$ & $-0,262$ & 0,069 & 0,408 & 0,601 & 0,940 & 0,670 & 0,592 & $-0, \times 93$ & 0,270 & 0,592 & $-0,193$ \\
\hline VI & $0,53^{2}$ & 0,638 & 0,767 & 0,253 & 1,106 & 0,592 & 0,468 & 0,339 & $-0,106$ & 0,514 & 0,129 & $-0,106$ & $0,5 \mathrm{I} 4$ \\
\hline VII & $-0,638$ & $-0,9 \mathrm{I} \mathrm{I}$ & $|-0,632|$ & $-0,562$ & 0,727 & 0,797 & 1,638 & I, 359 & 0,273 & $-0,070$ & 0,279 & 0,273 & $-0,070$ \\
\hline VIII & 0,911 & 1) 0,837 & 0,761 & 0,675 & 0,926 & 0,840 & 0,089 & 0,165 & 0,074 & 0,086 & $-0,076$ & 0,074 & 0,086 \\
\hline IX & $-0,837$ & $-0,734$ & $-0,498$ & $-0,7 \mathrm{I} 6$ & $\mathrm{I}, \mathrm{IO}_{3}$ & 0,885 & 1,837 & I, 601 & $-0,103$ & 0,218 & 0,236 & $-0,103$ & $0,2 \times 8$ \\
\hline $\mathrm{X}$ & 0,734 & $\begin{array}{l}0,439 \\
+\quad 0\end{array}$ & 0,318 & $0,6 \pm 7$ & 0,705 & $\mathrm{I}, 004$ & 0,266 & 0,387 & 0,295 & $-0,299$ & $-0,121$ & 0,295 & $-0,3^{\circ} 3$ \\
\hline XI & $-0,439$ & $-0,101$ & 0,018 & $-0,409$ & $\pi, 338$ & $0,9 \times 3$ & 1,439 & 1,322 & $-0,338$ & 0,425 & 0,117 & $-0,340$ & 0,425 \\
\hline XII & 0,101 & $-0,262$ & $-0,290$ & 0,142 & 0,637 & $1,07 \mathrm{I}$ & 0,899 & 0,929 & $0,36_{3}$ & $-0,434$ & $-0,030$ & 0,367 & $-0,434$ \\
\hline XIII & 0,262 & 0,579 & 0,538 & $0,16 \mathrm{r}$ & 1,317 & 0,940 & $0,73^{8}$ & 0,779 & 0,317 & 0,377 & $-0,04 \mathrm{I}$ & $-0,315$ & 0,377 \\
\hline XIV & $-0,579$ & $-0,785$ & $-0,68_{4}$ & $-0,421$ & 0,794 & 1,057 & 1,579 & $\mathrm{I}, 478$ & 0,206 & $-0,263$ & 0,101 & 0,206 & $-0,263$ \\
\hline$X V$ & 0,785 & 0,863 & 0,705 & $0,6 \mathrm{I} 7$ & 1,078 & 0,990 & 0,215 & 0,373 & $-0,078$ & 0,088 & $-0,158$ & $-0,078$ & 0,088 \\
\hline Spalte $\mathrm{Nr}$ & I & 2 & 3 & 4 & 5 & 6 & 7 & 8 & 9 & IO & II & I 2 & I 3 \\
\hline
\end{tabular}

Tabelle II. Die Spule mit großer Streuung: $\sigma=0,6, r=w$.

(Die Schaltspannung $\mathbf{E}$ ist gleich I gesetzt.)

\begin{tabular}{|c|c|c|c|c|c|c|c|c|c|c|c|c|c|}
\hline \multirow{3}{*}{$\begin{array}{c}\begin{array}{c}\text { Ordnungs- } \\
\text { zahl des } \\
\text { Intervalles }\end{array} \\
\mathrm{p}\end{array}$} & \multicolumn{4}{|c|}{ Wanderwellen } & \multirow{2}{*}{\multicolumn{2}{|c|}{$\begin{array}{l}\text { Knoten- } \\
\text { punkts- } \\
\text { potentiale }\end{array}$}} & \multirow{2}{*}{\multicolumn{2}{|c|}{$\begin{array}{l}\text { Lade- } \\
\text { zustand }\end{array}$}} & \multicolumn{5}{|c|}{ Windungsspannung } \\
\hline & \multicolumn{2}{|c|}{$\begin{array}{l}\text { Anfangs- } \\
\text { windung }\end{array}$} & \multicolumn{2}{|c|}{ Endwindung } & & & & & \multicolumn{2}{|c|}{ Vorderseite } & Rücken & \multicolumn{2}{|c|}{ 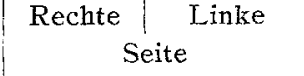 } \\
\hline & $V_{1}^{\prime}$ & $\mathrm{V}_{2}^{\prime \prime}$ & $\mathrm{V}_{2}^{\prime}$ & $\mathrm{V}_{3}^{\prime \prime}$ & $\mathrm{V}_{\mathrm{g}}$ & $\mathrm{V}_{3}$ & $V_{10}$ & $\mathrm{~V}_{30}$ & $E-V_{2}$ & $\mathrm{~V}_{2}-\mathrm{V}_{3}$ & $\mid \begin{array}{c}\bar{V}_{10} \\
-V_{30}\end{array}$ & $\begin{array}{r}V_{10}-V_{30} \\
+V_{1}^{\prime}-V_{2}^{\prime}\end{array}$ & $\begin{array}{r}V_{10}-V_{30} \\
+V_{2}^{\prime \prime}-V_{3}^{\prime \prime}\end{array}$ \\
\hline I & $\mathbf{I}$ & $0,36 \mathrm{I}$ & 0,361 & 0.143 & 0,361 & 0,143 & 0 & 0 & 0,639 & 0,218 & 0 & 0,639 & 0,218 \\
\hline II & $-0,36 t$ & $-0,338$ & 0,519 & $-0,438$ & 1,023 & 0,076 & $\mathrm{I}, 36 \mathrm{I}$ & 0,504 & $-0,023$ & 0,947 & 0,857 & $-0,023$ & 0,957 \\
\hline III & 0,338 & $\mid-0,345$ & $\mid \begin{array}{r}0,319 \\
-0,268\end{array}$ & 0,136 & 0,317 & $0,62 \mathrm{I}$ & 0,662 & 0,585 & 0,683 & $-0,304$ & 0,077 & 0,683 & $-0,404$ \\
\hline IV & 0,345 & $; \quad 0,428$ & 0,630 & $-0,031$ & 1,083 & 0,422 & 0,655 & 0,453 & $-0,083$ & $0,66 \mathrm{I}$ & 0,202 & $-0,083$ & $0,66 \mathrm{I}$ \\
\hline $\mathrm{V}$ & $\begin{array}{r}1,247 \\
-0,428\end{array}$ & $-0,555$ & $-0,189$ & $-0,202$ & 0,863 & 0,850 & 1,428 & 1,052 & 0,137 & $0,0 I_{3}$ & 0,376 & 0,137 & 0,013 \\
\hline VI & 0,565 & 0,290 & 0,064 & 0,237 & 0,725 & 0,898 & 0,435 & $0,66 \mathrm{I}$ & 0,275 & 0,173 & $-0,226$ & 0,275 & $-0,173$ \\
\hline VII & $-0,290$ & $-0,037$ & $0,29 I$ & $-0,222$ & $\mathrm{I}, 253$ & 0,740 & 1,290 & 0,962 & $|-0,253|$ & $0,5 \mathrm{I} 3$ & 0,328 & $-0,253$ & 0,513 \\
\hline VIII & 0,037 & $-0,264$ & $-0,332$ & 0,083 & 0,699 & 1,114 & 0,963 & $I, 031$ & $0,30 \mathrm{I}$ & $-0,445$ & $-0,068$ & 0,301 & $-0,415$ \\
\hline IX & 0,264 & $\begin{array}{r}0,370 \\
+\quad 0\end{array}$ & $\begin{array}{l}0,324 \\
0\end{array}$ & 0,048 & $1, x 06$ & 0,830 & 0,736 & 0,782 & $-0,106$ & 0,276 & $-0,046$ & $-0,106$ & 0,276 \\
\hline $\mathrm{X}$ & $-0,370$ & $-0,338$ & $-0,122$ & $-0,156$ & $1,03^{2}$ & 0,998 & 1,370 & I, I 54 & $-0,032$ & 0,034 & 0,2 I 6 & $-0,032$ & 0,034 \\
\hline $\mathrm{XI}$ & 0,338 & 0,151 & $-0,063$ & 0,175 & 0,813 & $1,05 \mathrm{I}$ & 0,662 & 0,876 & 0,187 & $-0,23^{8}$ & $-0,214$ & 0,187 & $-0,238$ \\
\hline XII & $-0,15 \mathrm{I}$ & 0,060 & 0,223 & $-0,125$ & $\mathrm{I}, 2 \mathrm{I} \mathrm{I}$ & 0,863 & $\mathrm{I}, \mathrm{X} 5 \mathrm{I}$ & 0,988 & $-0,2$ II & 0,348 & $0,163 \mid$ & $-0,211$ & 0,348 \\
\hline XIII * & $-0,060$ & $-0,225$ & $-0,25 \mathrm{I}$ & 0,026 & 0,835 & $1,1 \mathrm{I} 3$ & 1,060 & 1,086 & 0,165 & $3-0,278$ & $-0,026$ & $-0,165$ & $-0,277$ \\
\hline XIV & 0,225 & 0,269 & 0,183 & 0,067 & 1,044 & 0,928 & 0,775 & $0,86 \mathrm{I}$ & $-0,044$ & 0, I 16 & $-0,086$ & $-0,044$ & 0,116 \\
\hline$X V$ & $-0,260$ & $\begin{array}{l}0,129 \\
-0,199\end{array}$ & $-0,04 \mathrm{I}$ & $-0,121$ & I,070 & 0,990 & 1,269 & $\mathrm{I}, \mathrm{I} I \mathrm{I}$ & $-0,070$ & 0,080 & 0,158 & $-0,070$ & 0,080 \\
\hline $\mathrm{XVI}$ & 0,199 & 9. 0,055 & $-0,093$ & $0,1 \times 7$ & 0,856 & 1,066 & $0,80 \mathrm{I}$ & 0,949 & 0,144 & $-0,210$ & $-0,148$ & 0,144 & $-0,210$ \\
\hline Spa & I & 2 & 3 & 4 & 5 & 6 & 7 & 8 & 9 & 10 & II & 12 & 13 \\
\hline
\end{tabular}

Ebenfalls leicht anzugeben ist die Windungsspannung in der Mitte der Spulenrïckseite. Da hier die rechts- und dinksläufigen Wellen gleichzeitig eintreffen, sind $V_{10}$ bzw. $V_{30}$ (Abb. I4 a und $b$ ) die Werte des Potentials. Thre Differenz, die Windungsspannung, ist in Spalte II aufgeführt. Die Maximalwerte $0,5 \mathrm{E}$ bzw. $0,86 \mathrm{E}$ liegen etwas tiefer als die Windungsspannungen auf der Vorderseite. 

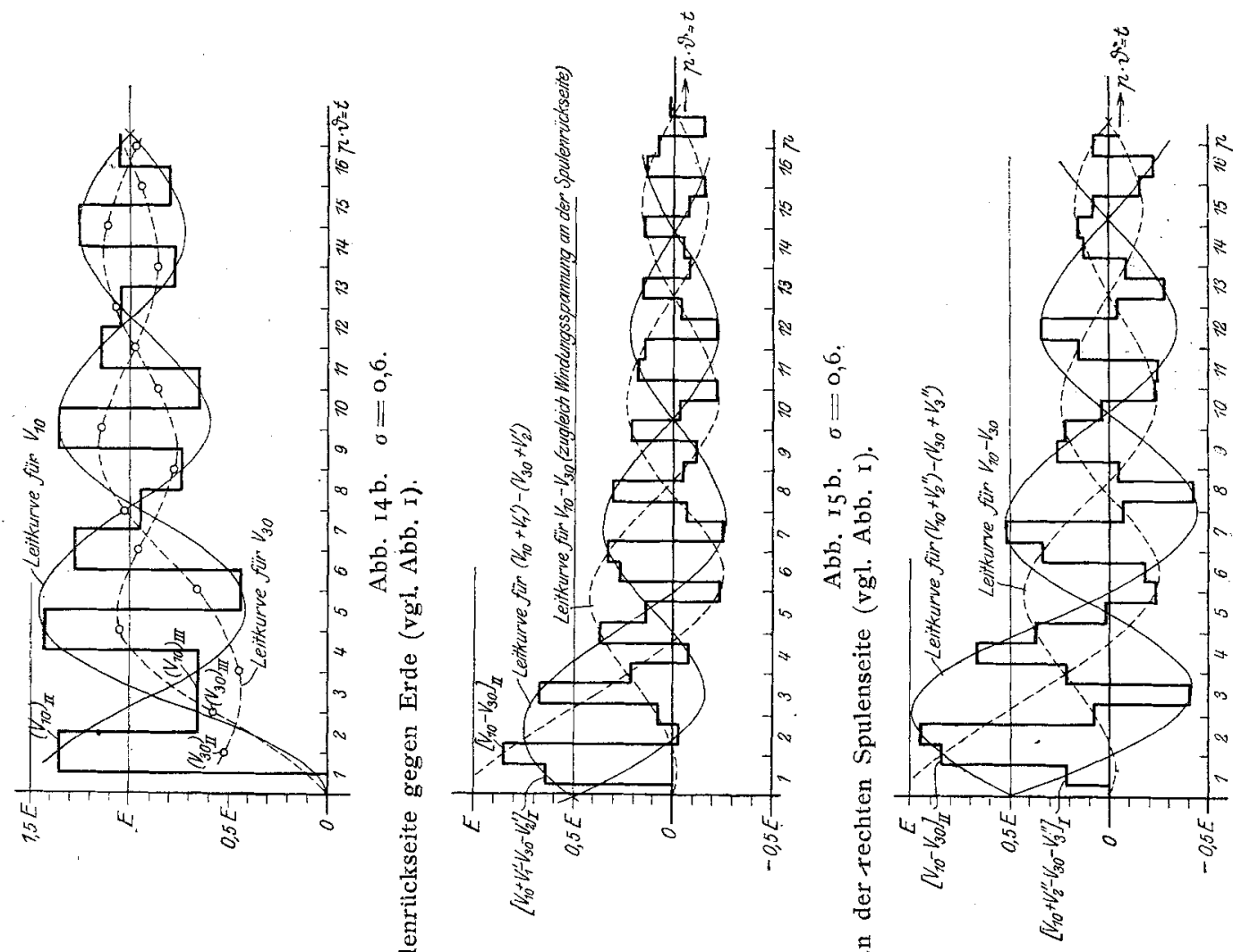

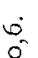

$\dot{2}$ $\dot{0}$

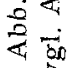
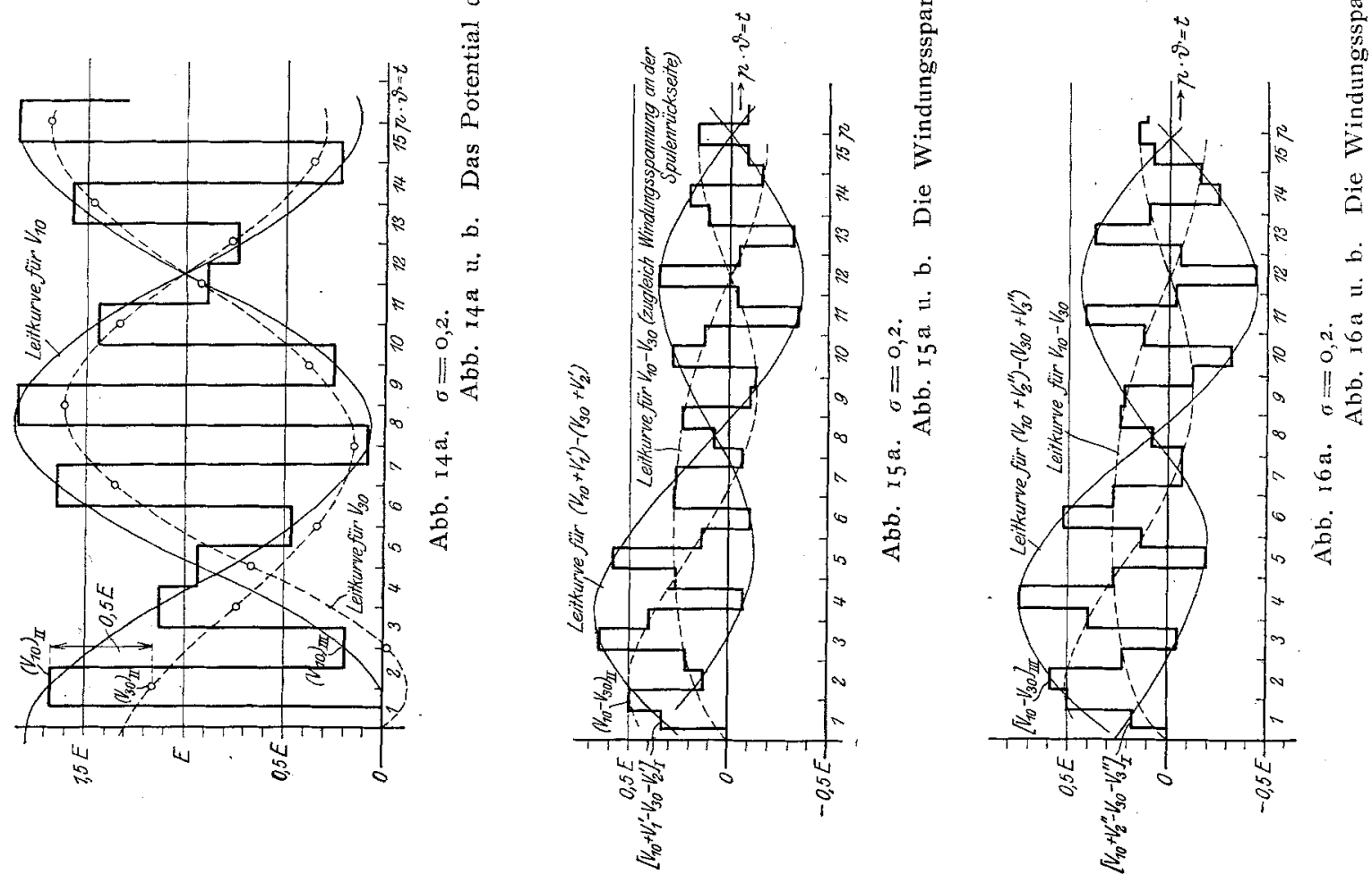
SchlieBlich zeigen noch die Abb. I5 und 16 die eigenartige Pulsation der Windungsspannung in der Mitte der beiden Spulenseiten (vgl. auch Abb. I). Es treffen hier auf ein Intervall zwei Stufen, da die Ankunftszeit der rechtsläufigen und linksläufigen Welle um ein halbes Intervall differiert. Gleich nach dem Einschalten begegnen wir wieder denselben gefährlich hohen Werten, wie an der Vorderseite der Spule, wobei abermals auf die Endwindung eine etwas höhere Beanspruchung entfällt. Ist jedoch die Anfangsschwingung abgeklungen, so steigen die Spitzen nicht mehr über die halbe Einschaltspannung.

Gegen Erde wird die ganze Spule mit hoher Spannung beansprucht, am höchsten die erste Windung $\left(\mathrm{V}_{\mathbf{1 0}} \mathrm{l}\right)$ der Spule mit geringer Streuung, wo nicht viel an der Erreichung der doppelten Schaltspannung $2 \mathrm{E}$ fehlt. 